\title{
A superprocess involving both branching and coalescing
}

\author{
Xiaowen Zhou ${ }^{1}$ \\ Department of Mathematics and Statistics, Concordia University, 1455 de Maisonneuve Blvd. West, Montreal, Quebec, H3G 1M8, Canada
}

Received 5 April 2005; received in revised form 11 April 2006; accepted 12 September 2006

Available online 12 January 2007

\begin{abstract}
We consider a superprocess with coalescing Brownian spatial motion. We first point out a dual relationship between two systems of coalescing Brownian motions. In consequence we can express the Laplace functionals for the superprocess in terms of coalescing Brownian motions, which allows us to obtain some explicit results. We also point out several connections between such a superprocess and the Arratia flow. A more general model is discussed at the end of this paper.
\end{abstract}

๔) 2007 Elsevier Masson SAS. All rights reserved.

\section{Résumé}

Nous considérons un super-processus avec mouvement Brownien spatial coalescent. Nous soulignons d'abord une relation de dualité entre deux systèmes de mouvements Browniens coalescents. Il en résulte une expression des fonctionnelles de Laplace pour ces super-processus en termes de mouvements Browniens coalescents, ce qui nous permet d'obtenir certains résultats explicites. Nous soulignons aussi plusieurs relations entre un tel super-processus et le flot d'Arratia. Un modèle plus général est discuté en conclusion d'article.

(c) 2007 Elsevier Masson SAS. All rights reserved.

MSC: 60G57; 60J65; 60G80; 60K35

Keywords: Coalescing simple random walk; Coalescing Brownian motion; Duality; Superprocess with coalescing Brownian spatial motion;

Arratia flow; Laplace functional; Feller's branching diffusion; Bessel process

\section{Introduction}

In this paper we mainly consider the following branching-coalescing particle system which can be described intuitively as follows. A collection of particles with masses execute coalescing Brownian motions. Meanwhile the masses for these particles evolve according to independent Feller's branching diffusions. Upon coalescing the two particles involved merge together to one particle where the mass of the new particle is the sum of the masses of the coalesced particles.

The above-mentioned particle system can be described using a measure-valued process $Z$. More precisely, the support of $Z_{t}$ represents the locations of the particles at time $t$, and the measure $Z_{t}$ assigned to each point in its support stands for the mass of the corresponding particle. This process $Z$, which we call the superprocess with coalescing

E-mail address: zhou@alcor.concordia.ca.

1 The author is supported by an NSERC grant. 
Brownian spatial motion (SCSM), was first introduced in [5]. It arises as a scaling limit of another measure-valued process, which was referred to in [4] as the superprocess with dependent spatial motion (SDSM). SDSM arises as a high density limit of a critical branching particle system in which the motion of each particle is subjected to both an independent Brownian motion and a common white noise applied to all the particles. More precisely, the movement of the $i$ th particle is governed by equation

$$
\mathrm{d} x_{i}(t)=\sigma\left(x_{i}(t)\right) \mathrm{d} B_{i}(t)+\int_{\mathbb{R}} h\left(y-x_{i}(t)\right) W(\mathrm{~d} y, \mathrm{~d} t),
$$

where $\left(B_{i}\right)$ is a collection of independent Brownian motions which is independent of the white noise $W$; see [4]. A similar model was also studied in [14].

It was shown in Theorem 4.2 of [5] that, after appropriate time-space scaling, SDSM converges weakly to SCSM. A functional dual for SCSM was given in Theorem 3.4 of [5]. In addition, using coalescing Brownian motions and excursions for Feller's branching diffusion, a construction of SCSM was found in [5], an idea that initially came from [3]. In this paper we always denote such a SCSM as Z.

One of the most interesting problems in the study of a measure-valued process is to recover a certain duality relation concerning the measure-valued process. Such a dual relationship often leads to the uniqueness of the measure-valued process; see [12] for some classical examples on super Brownian motion and related processes. It is not hard to show the existence of $Z$ as a high density limit of the branching-coalescing particle systems. The main goal of this paper is to propose a new way of characterizing the measure-valued process $Z$ via duality, in which the self-duality for coalescing Brownian motions plays a key role. To this end, we first point out a rather general duality on two coalescing Brownian motions running in the opposite directions. With this duality we can express certain Laplace functionals for $Z$ in terms of systems of coalescing Brownian motions.

We could carry out some explicit computation thanks to the above-mentioned duality. In particular, we first show that, starting with a possibly diffuse initial finite measure $Z_{0}, Z_{t}$ collapses into a discrete measure with a finite support as soon as $t>0$. Then we can identify $Z_{t}$ interchangeably with a finite collection of spatially distributed particles with masses. When there is such a particle at a fixed location, we obtain the Laplace transform of its mass. The total number of particles in $Z_{t}$ decreases in $t$ due to both branching and coalescing. When there is only one particle left at time $t$, we can recover the joint distribution of its location and its mass. Eventually, all the particles will die out. We further find the distribution of the location where the last particle disappears. Coincidentally, super Brownian motion shares the same near extinction behavior.

Connections between superprocesses and stochastic flows have been noticed before. For instance, in [11] a superprocess was obtained from the empirical measure of a coalescing flow. Arratia flow serves as a fundamental example of a coalescing flow. In this paper we point out several connections between $Z$ and the Arratia flow. More precisely, the support of $Z_{t}$ at a fixed time $t>0$ can be identified with a Cox process whose intensity measure is determined by the Arratia flow. A version of $Z_{t}$ can be constructed using the Arratia flow. The general Laplace functional for $Z$ can also be expressed in terms of the Arratia flow.

Replacing the Feller's branching diffusion by the square of the Bessel process (BESQ) to incorporate immigration, we introduce and discuss a more general model at the end of this paper. Since dimension is a parameter for BESQ representing the immigration rate, in addition to the measure-valued process for the mass, in the modified model we introduce another measure-valued process to describe the dimension. The simultaneous mass-dimension evolution of such a model can also be characterized by coalescing Brownian motions.

The rest of this paper is arranged as follows. As a preliminary, we first state and prove a duality relation on coalescing Brownian motions in Section 2. In Section 3, we define the process $Z$ as a weak limit of the empirical measures for the branching-coalescing particle systems. Then we proceed to prove the duality between $Z$ and coalescing Brownian motions. The uniqueness of $Z$ follows from such a duality immediately. We continue to study several properties of this process in Section 4. We further discuss the connections between the Arratia flow and $Z$ in Section 5. At the end of this paper, we propose a more general model and establish its duality in Section 6.

\section{Coalescing Brownian motions and their duality}

An $m$-dimensional coalescing Brownian motion can be described as follows. Consider a system of $m$ indexed particles with locations in $\mathbb{R}$ that evolves as follows. Each particle moves according to an independent standard Brownian 
motion on $\mathbb{R}$ until two particles are at the same location. At this moment a coalescence event occurs and the particle of higher index starts to move together with the particle of lower index. We say the particle with higher index is attached to the particle with lower index, which is still free. The particle system then continues its evolution in the same fashion. Note that indices are not essential here, the collection of locations of the particles is Markovian in its own right, but it will be convenient to think of the process as taking values in $\mathbb{R}^{m}$ rather than subsets of $\mathbb{R}$ with at most $m$ elements. For definiteness, throughout this section we will further assume that the particles are indexed in increasing order of their initial positions: it is clear that the dynamics preserve this ordering. Call the resulting Markov process $\mathbf{X}=\left(X_{1}, \ldots, X_{m}\right)$.

Write $1\{B\}(\cdot)$ for the indicator function of a set $B$. The distribution of $\mathbf{X}(t)$ is uniquely specified by knowing for each choice of $y_{1}<y_{2}<\cdots<y_{n}$ the joint probabilities of which "balls" $X_{1}(t), X_{2}(t), \ldots, X_{m}(t)$ lie in which of the "boxes" $\left.\left.\left.\left.\left.] y_{1}, y_{2}\right],\right] y_{2}, y_{3}\right], \ldots,\right] y_{n-1}, y_{n}\right]$. That is, the distribution of $\mathbf{X}(t)$ is determined by the joint distribution of the indicators

$$
\left.\left.I_{i j}(t, \mathbf{y}):=1\left\{X_{i}(t) \in\right] y_{j}, y_{j+1}\right]\right\}
$$

for $1 \leqslant i \leqslant m, 1 \leqslant j \leqslant n-1$ and $\mathbf{y}=\left(y_{1}, \ldots, y_{n}\right)$.

Suppose now that $\mathbf{Y}:=\left(Y_{1}, \ldots, Y_{n}\right)$ is another coalescing Brownian motion. The distribution of $\mathbf{Y}(t)$ is uniquely specified by knowing for each choice of $x_{1}<x_{2}<\cdots<x_{n}$ the distribution of the indicators

$$
\left.\left.I_{i j}^{\leftarrow}(t, \mathbf{x}):=1\left\{x_{i} \in\right] Y_{j}(t), Y_{j+1}(t)\right]\right\}
$$

for $1 \leqslant i \leqslant m, 1 \leqslant j \leqslant n-1$ and $\mathbf{x}=\left(x_{1}, \ldots, x_{m}\right)$.

The next "balls-in-boxes" duality is crucial in characterizing the distributions of the measure-valued processes considered in this paper.

Theorem 2.1. Suppose in the notation above that $\mathbf{X}=\left(X_{1}, \ldots, X_{m}\right)$ is an $m$-dimensional coalescing Brownian motion and $\mathbf{Y}=\left(Y_{1}, \ldots, Y_{n}\right)$ is an $n$-dimensional coalescing Brownian motion. Then for each $t \geqslant 0$ the joint distribution of the $m \times(n-1)$-dimensional random array $\left(I_{i j}(t, \mathbf{Y}(0))\right)$ coincides with that of the $m \times(n-1)$-dimensional random $\operatorname{array}\left(I_{i j}^{\leftarrow}(t, \mathbf{X}(0))\right)$.

Theorem 2.1 can be seen from Theorem 8 of [15], which concerns a more elaborate duality on a system of Brownian motions. In such a system, some Brownian motions run forwards in time, and the others run backwards in time. Those Brownian motions running in the same direction coalesce whenever they meet, and those running in the opposite direction reflect on each other. Theorem 8 of [15] shows that the order of coalescing and reflecting does not change the joint distribution of the system. Other dualities on coalescing-reflecting Brownian systems can also be found in [16].

In this paper we will give a direct proof of the "balls-in-boxes" duality. We first prove the counterpart of Theorem 2.1 for continuous time simple coalescing random walks, which is interesting in its own right. Notice that $\mathbf{X}$ is a coalescing Brownian motion if and only if $X_{i}$ is a $\left(\mathcal{F}_{t}^{\mathbf{X}}\right)$-Brownian motion for each $1 \leqslant i \leqslant m$, and $\left(X_{j}-X_{i}\right) / \sqrt{2}$ is a $\left(\mathcal{F}_{t}^{\mathbf{X}}\right)$-Brownian motion stopped at 0 , where $\left(\mathcal{F}_{t}^{\mathbf{X}}\right)$ denotes the filtration generated by $\mathbf{X}$. Then Theorem 2.1 follows from a straight forward martingale argument proof for the convergence of the scaled random walk to Brownian motion.

For discrete time simple coalescing random walks the "balls-in-boxes" duality is evident from Fig. 7 of [15]. But the duality seems to be less apparent for continuous time simple coalescing random walks.

A $p$-simple random walk on $\mathbb{Z}$ is a continuous time simple random walk that makes jumps at unit rate, and when it makes a jump from a certain site it jumps to the right neighbor with probability $p$ and to the left neighbor with probability $1-p$. An $m$-dimensional $p$-simple coalescing random walk is defined in the same way as the coalescing Brownian motion at the beginning of this section. When $p=1 / 2$ we just call this particle system a simple coalescing random walk.

Some notation is useful to keep track of the interactions among the particles in the coalescing system. Let $\mathcal{P}_{m}$ denote the set of interval partitions of the totality of indices $\mathbb{N}_{m}:=\{1, \ldots, m\}$. That is, an element $\pi$ of $\mathcal{P}_{m}$ is a collection $\pi=\left\{A_{1}(\pi), \ldots, A_{h}(\pi)\right\}$ of disjoint subsets of $\mathbb{N}_{m}$ such that $\bigcup_{i} A_{i}(\pi)=\mathbb{N}_{m}$ and $a<b$ for all $a \in A_{i}$, $b \in A_{j}, i<j$. The sets $A_{1}(\pi), \ldots, A_{h}(\pi)$ consisting of consecutive indices are the intervals of the partition $\pi$. The integer $h$ is the length of $\pi$ and is denoted by $l(\pi)$. Equivalently, we can think of $\mathcal{P}_{m}$ as a set of equivalence relations 
on $\mathbb{N}_{m}$ and write $i \sim_{\pi} j$ if $i$ and $j$ belong to the same interval of $\pi \in \mathcal{P}_{m}$. Of course, if $i \sim_{\pi} j$, then $i \sim_{\pi} k \sim_{\pi} j$ for all $i \leqslant k \leqslant j$.

Now we want to introduce the state space for the simple coalescing random walk. Given $\pi \in \mathcal{P}_{m}$, define

$$
\alpha_{i}(\pi):=\min A_{i}(\pi)
$$

to be the left-hand end-point of the $i$ th interval $A_{i}(\pi)$. Put

$$
\mathbb{Z}_{\pi}^{m}:=\left\{\left(x_{1}, \ldots, x_{m}\right) \in \mathbb{Z}^{m}: x_{1} \leqslant \cdots \leqslant x_{m} \text { and } x_{i}=x_{j} \text { if } i \sim_{\pi} j\right\}
$$

and

$$
\widehat{\mathbb{Z}}_{\pi}^{m}:=\left\{\left(x_{1}, \ldots, x_{m}\right) \in \mathbb{Z}^{m}: x_{1} \leqslant \cdots \leqslant x_{m} \text { and } x_{i}=x_{j} \text { if and only if } i \sim_{\pi} j\right\} .
$$

Note that $\mathbb{Z}^{m}$ is the disjoint union of the sets $\widehat{\mathbb{Z}}_{\pi}^{m}, \pi \in \mathcal{P}_{m}$.

Write $\mathbf{X}=\left(X_{1}, \ldots, X_{m}\right)$ for the $p$-simple coalescing random walk. If $\mathbf{X}(t) \in \widehat{\mathbb{Z}}_{\pi}^{m}$, then the free particles at time $t$ have indices $\alpha_{1}(\pi), \ldots, \alpha_{l(\pi)}(\pi)$ and the $i$ th particle at time $t$ is attached to the free particle with index

$$
\min \left\{j: 1 \leqslant j \leqslant m, j \sim_{\pi} i\right\}=\max \left\{\alpha_{k}(\pi): \alpha_{k}(\pi) \leqslant i\right\} .
$$

In order to write down the generator for $\mathbf{X}$, we require a final piece of notation. Let $\left\{\mathbf{e}_{i}^{k}: 1 \leqslant i \leqslant k\right\}$ be the set of coordinate vectors in $\mathbb{Z}^{k}$; that is, $\mathbf{e}_{i}^{k}$ is the vector that has the $i$ th coordinate 1 and all the other coordinates 0 . For $\pi \in \mathcal{P}_{m}$, define a map $K_{\pi}: \mathbb{Z}_{\pi}^{m} \rightarrow \mathbb{Z}^{l(\pi)}$ by

$$
K_{\pi}(\mathbf{x})=K_{\pi}\left(x_{1}, \ldots, x_{m}\right):=\left(x_{\alpha_{1}(\pi)}, \ldots, x_{\alpha_{l(\pi)}(\pi)}\right) .
$$

Notice that $K_{\pi}$ is a bijection between $\mathbb{Z}_{\pi}^{m}$ and $\left\{x \in \mathbb{Z}^{l(\pi)}: x_{1} \leqslant x_{2} \leqslant \cdots \leqslant x_{l(\pi)}\right\}$, and we write $K_{\pi}^{-1}$ for the inverse of $K_{\pi}$. For brevity, we will sometimes write $\mathbf{x}_{\pi}$ for $K_{\pi}(\mathbf{x})$.

Write $B\left(\mathbb{Z}^{m}\right)$ for the collection of all bounded functions on $\mathbb{Z}^{m}$. The generator $G$ for $\mathbf{X}$ is the operator $G: B\left(\mathbb{Z}^{m}\right) \rightarrow$ $B\left(\mathbb{Z}^{m}\right)$ given by

$$
\begin{aligned}
& G f(\mathbf{x}):=p \sum_{i=1}^{l(\pi)} f \circ K_{\pi}^{-1}\left(\mathbf{x}_{\pi}+\mathbf{e}_{i}^{l(\pi)}\right)+(1-p) \sum_{i=1}^{l(\pi)} f \circ K_{\pi}^{-1}\left(\mathbf{x}_{\pi}-\mathbf{e}_{i}^{l(\pi)}\right)-l(\pi) f \circ K_{\pi}^{-1}\left(\mathbf{x}_{\pi}\right), \\
& f \in B\left(\mathbb{Z}^{m}\right), \mathbf{x} \in \widehat{\mathbb{Z}}_{\pi}^{m}, \pi \in \mathcal{P}_{m} .
\end{aligned}
$$

This expression is well-defined, because if $\mathbf{x} \in \widehat{\mathbb{Z}}_{\pi}^{m}$, then $\mathbf{x}_{\pi}, \mathbf{x}_{\pi}+\mathbf{e}_{i}^{l(\pi)}$ and $\mathbf{x}_{\pi}-\mathbf{e}_{i}^{l(\pi)}$ are all in $\left\{x \in \mathbb{Z}^{l(\pi)}: x_{1} \leqslant x_{2} \leqslant\right.$ $\left.\cdots \leqslant x_{l(\pi)}\right\}$.

Note. From now on we will suppress the dependence on dimension and write $\mathbf{e}_{i}^{l(\pi)}$ as $\mathbf{e}_{i}$.

Write $\mathbb{Z}^{\prime}:=\mathbb{Z}+1 / 2=\{i+1 / 2: i \in \mathbb{Z}\}$. An $n$-dimensional $q$-simple coalescing random walk on $\mathbb{Z}^{\prime n}$ and its generator $H$ can be defined in the obvious way. Such a process, with $q=1-p$, will serve as the process dual to the $p$-simple coalescing random walk on $\mathbb{Z}^{m}$ in the following way.

Fix $\mathbf{x} \in \mathbb{Z}^{m}$ with $x_{1} \leqslant \cdots \leqslant x_{m}$ and $\mathbf{y} \in \mathbb{Z}^{\prime n}$ with $y_{1} \leqslant \cdots \leqslant y_{n}$. Put

$$
\left.\left.I_{i j}(t, \mathbf{y}):=1\left\{X_{i}(t) \in\right] y_{j}, y_{j+1}\right]\right\}
$$

and

$$
\left.\left.I_{i j}^{\leftarrow}(t, \mathbf{x}):=1\left\{x_{i} \in\right] Y_{j}(t), Y_{j+1}(t)\right]\right\}
$$

for $1 \leqslant i \leqslant m$ and $1 \leqslant j \leqslant n-1$.

Lemma 2.2. Suppose in the notation above that $\mathbf{X}=\left(X_{1}, \ldots, X_{m}\right)$ is an $m$-dimensional $\mathbb{Z}^{m}$-valued $p$-simple coalescing random walk and $\mathbf{Y}=\left(Y_{1}, \ldots, Y_{n}\right)$ is an $n$-dimensional $\mathbb{Z}^{\prime n}$-valued $(1-p)$-simple coalescing random walk. Then for each $t \geqslant 0$ the joint distribution of the $m \times(n-1)$-dimensional random array $\left(I_{i j}(t, \mathbf{Y}(0))\right)$ coincides with that of the $m \times(n-1)$-dimensional random array $\left(I_{i j}^{\leftarrow}(t, \mathbf{X}(0))\right)$. 
Proof. For a bounded function $g:\{0,1\}^{m(n-1)} \rightarrow \mathbb{R}$, a vector $\mathbf{x} \in \mathbb{Z}^{m}$ with $x_{1} \leqslant \cdots \leqslant x_{m}$, and a vector $\mathbf{y} \in \mathbb{Z}^{\prime n}$ with $y_{1} \leqslant \cdots \leqslant y_{n}$, set

$$
\left.\left.\left.\left.\left.\left.\left.\left.\bar{g}(\mathbf{x} ; \mathbf{y}):=g\left(1\{] y_{1}, y_{2}\right]\right\}\left(x_{1}\right), \ldots, 1\{] y_{n-1}, y_{n}\right]\right\}\left(x_{1}\right), \ldots, 1\{] y_{1}, y_{2}\right]\right\}\left(x_{m}\right), \ldots, 1\{] y_{n-1}, y_{n}\right]\right\}\left(x_{m}\right)\right) .
$$

We may assume that $\mathbf{X}$ and $\mathbf{Y}$ are defined on the same probability space $(\Omega, \mathcal{F}, \mathbb{P})$. We need to show that

$$
\mathbb{P}[\bar{g}(\mathbf{X}(t) ; \mathbf{Y}(0))]=\mathbb{P}[\bar{g}(\mathbf{X}(0) ; \mathbf{Y}(t))] .
$$

For $\mathbf{x} \in \mathbb{Z}^{m}$, put $\bar{g}_{\mathbf{x}}(\cdot):=\bar{g}(\mathbf{x} ; \cdot)$, and for $\mathbf{y} \in \mathbb{Z}^{\prime n}$, put $\bar{g}_{\mathbf{y}}(\cdot):=\bar{g}(\cdot ; \mathbf{y})$. In order to establish (2.1), it suffices by a standard argument (cf. Section 4.4 of [7]) to show that

$$
G\left(\bar{g}_{\mathbf{y}}\right)(\mathbf{x})=H\left(\bar{g}_{\mathbf{x}}\right)(\mathbf{y})
$$

(recall that $G$ and $H$ are the generators of $\mathbf{X}$ and $\mathbf{Y}$, respectively).

Fix $\mathbf{x} \in \widehat{\mathbb{Z}}_{\pi}^{m}$ and $\mathbf{y} \in \widehat{\mathbb{Z}}_{\varpi}^{\prime n}$ for some $\pi \in \mathcal{P}_{m}$ and $\varpi \in \mathcal{P}_{n}$. Put

$$
I^{+}:=\left\{i: 1 \leqslant i \leqslant l(\pi), x_{\alpha_{i}(\pi)}+1 / 2=y_{\alpha_{j}(\varpi)} \text { for some } 1 \leqslant j \leqslant l(\varpi)\right\}
$$

and

$$
I^{-}:=\left\{i: 1 \leqslant i \leqslant l(\pi), x_{\alpha_{i}(\pi)}-1 / 2=y_{\alpha_{j}(\varpi)} \text { for some } 1 \leqslant j \leqslant l(\varpi)\right\} .
$$

Similarly, put

$$
J^{-}:=\left\{j: 1 \leqslant j \leqslant l(\varpi), y_{\alpha_{j}(\varpi)}-1 / 2=x_{\alpha_{i}(\pi)} \text { for some } 1 \leqslant i \leqslant l(\pi)\right\}
$$

and

$$
J^{+}:=\left\{j: 1 \leqslant j \leqslant l(\varpi), y_{\alpha_{j}(\varpi)}+1 / 2=x_{\alpha_{i}(\pi)} \text { for some } 1 \leqslant i \leqslant l(\pi)\right\} .
$$

For each $i \in I^{+}$there is a unique $j \in J^{-}$such that $x_{\alpha_{i}(\pi)}+1 / 2=y_{\alpha_{j}(\varpi)}$ and vice versa. Fix such a pair $(i, j)$, we can verify that

$$
\bar{g}_{\mathbf{y}} \circ K_{\pi}^{-1}\left(\mathbf{x}_{\pi}+\mathbf{e}_{i}\right)=\bar{g}_{\mathbf{x}} \circ K_{\pi}^{-1}\left(\mathbf{y}_{\varpi}-\mathbf{e}_{j}\right)
$$

by considering all the possible scenarios. In addition, it is easy to see for $i^{\prime} \notin I^{+}$that

$$
\bar{g}_{\mathbf{y}} \circ K_{\pi}^{-1}\left(\mathbf{x}_{\pi}+\mathbf{e}_{i^{\prime}}\right)=\bar{g}_{\mathbf{y}} \circ K_{\pi}^{-1}\left(\mathbf{x}_{\pi}\right)
$$

and for $j^{\prime} \notin J^{-}$that

$$
\bar{g}_{\mathbf{x}} \circ K_{\varpi}^{-1}\left(\mathbf{y}_{\varpi}-\mathbf{e}_{j^{\prime}}\right)=\bar{g}_{\mathbf{x}} \circ K_{\varpi}^{-1}\left(\mathbf{y}_{\varpi}\right) .
$$

Similarly, for any $i \in I^{-}$there exists a unique $j \in J^{+}$such that $x_{\alpha_{i}(\pi)}-1 / 2=y_{\alpha_{j}(\varpi)}$ and vice versa. For such a pair $(i, j)$ we have

$$
\bar{g}_{\mathbf{y}} \circ K_{\pi}^{-1}\left(\mathbf{x}_{\pi}-\mathbf{e}_{i}\right)=\bar{g}_{\mathbf{x}} \circ K_{\pi}^{-1}\left(\mathbf{y}_{\varpi}+\mathbf{e}_{j}\right) .
$$

Furthermore, we see for $i^{\prime} \notin I^{-}$that

$$
\bar{g}_{\mathbf{y}} \circ K_{\pi}^{-1}\left(\mathbf{x}_{\pi}-\mathbf{e}_{i^{\prime}}\right)=\bar{g}_{\mathbf{y}} \circ K_{\pi}^{-1}\left(\mathbf{x}_{\pi}\right)
$$

and for $j^{\prime} \notin J^{+}$that

$$
\bar{g}_{\mathbf{x}} \circ K_{\varpi}^{-1}\left(\mathbf{y}_{\varpi}+\mathbf{e}_{j^{\prime}}\right)=\bar{g}_{\mathbf{x}} \circ K_{\varpi}^{-1}\left(\mathbf{y}_{\varpi}\right) .
$$

Lastly, note that

$$
\bar{g}_{\mathbf{y}} \circ K_{\pi}^{-1}\left(\mathbf{x}_{\pi}\right)=\bar{g}(\mathbf{x} ; \mathbf{y})=\bar{g}_{\mathbf{x}} \circ K_{\varpi}^{-1}\left(\mathbf{y}_{\varpi}\right)
$$

and so 


$$
\begin{aligned}
G\left(\bar{g}_{\mathbf{y}}\right)(\mathbf{x})-H\left(\bar{g}_{\mathbf{x}}\right)(\mathbf{y})= & p \sum_{i \in I^{+}}\left(\bar{g}_{\mathbf{y}} \circ K_{\pi}^{-1}\left(\mathbf{x}_{\pi}+\mathbf{e}_{i}\right)-\bar{g}_{\mathbf{y}} \circ K_{\pi}^{-1}\left(\mathbf{x}_{\pi}\right)\right) \\
& +(1-p) \sum_{i \in I^{-}}\left(\bar{g}_{\mathbf{y}} \circ K_{\pi}^{-1}\left(\mathbf{x}_{\pi}-\mathbf{e}_{i}\right)-\bar{g}_{\mathbf{y}} \circ K_{\pi}^{-1}\left(\mathbf{x}_{\pi}\right)\right) \\
& -p \sum_{j \in J^{-}}\left(\bar{g}_{\mathbf{x}} \circ K_{\varpi}^{-1}\left(\mathbf{y}_{\varpi}-\mathbf{e}_{i}\right)-\bar{g}_{\mathbf{x}} \circ K_{\varpi}^{-1}\left(\mathbf{y}_{\varpi}\right)\right) \\
& -(1-p) \sum_{j \in J^{+}}\left(\bar{g}_{\mathbf{x}} \circ K_{\varpi}^{-1}\left(\mathbf{y}_{\varpi}+\mathbf{e}_{i}\right)-\bar{g}_{\mathbf{x}} \circ K_{\varpi}^{-1}\left(\mathbf{y}_{\varpi}\right)\right) \\
= & 0,
\end{aligned}
$$

as required.

\section{Existence and uniqueness}

A construction of $Z$ was given in [5] using Feller's branching excursions. In this paper we adopt a weak convergence approach, which is commonly used in the study of measure-valued processes.

Recall that a nonnegative valued process $\xi$ is a Feller's branching diffusion with initial value $x \geqslant 0$ if it is the unique strong solution to the following stochastic differential equation

$$
\xi(t)=x+\int_{0}^{t} \sqrt{\gamma \xi(s)} \mathrm{d} B(s),
$$

where $\gamma$ is a positive constant and $B$ is a one-dimensional Brownian motion. $(\xi(t))_{t \geqslant 0}$ is a martingale. Its onedimensional marginal can be characterized by its Laplace transform

$$
\mathbb{P}[\exp \{-\lambda \xi(t)\}]=\exp \left\{-\frac{2 \lambda x}{2+\lambda \gamma t}\right\}
$$

its extinction probability is then given by

$$
\mathbb{P}\{\xi(t)=0\}=\exp \left\{-\frac{2 x}{\gamma t}\right\}
$$

see Sections II.1 and II.5 of [12].

Observe that independent Feller's branching diffusions are additive; i.e. if $\xi$ and $\eta$ are two independent Feller's branching diffusions (with the same parameter $\gamma$ ), then $\xi+\eta$ is also a Feller's branching diffusion. This fact will be used repeatedly in our discussions.

Write $M_{F}(\mathbb{R})$ for the space of finite measures on $\mathbb{R}$ equipped with the topology of weak convergence. For any $\mu \in M_{F}(\mathbb{R})$ and any real valued function $f$ on $\mathbb{R}$, put

$$
\langle\mu, f\rangle=\int_{-\infty}^{\infty} f(x) \mu(\mathrm{d} x) .
$$

Given $Z_{0} \in M_{F}(\mathbb{R})$, put $\bar{z}:=Z_{0}(\mathbb{R})$. For any positive integer $m$, let $\left(\xi_{1}^{(m)}, \ldots, \xi_{m}^{(m)}\right)$ be a collection of $m$ independent Feller's branching diffusions each with initial value $\bar{z} / m$. Choose $\left(x_{1}, \ldots, x_{m}\right)$ to be i.i.d. samples from distribution $Z_{0} / \bar{z}$. Let $\left(X_{1}^{(m)}, \ldots, X_{m}^{(m)}\right)$ be an $m$-dimensional coalescing Brownian motion starting at $\left(x_{1}, \ldots, x_{m}\right)$. Moreover, we always assume that $\left(\xi_{i}^{(m)}\right)$ and $\left(X_{i}^{(m)}\right)$ are independent. Let $\delta_{x}$ denote the point mass at $x \in \mathbb{R}$. Then

$$
Z_{t}^{(m)}:=\sum_{i=1}^{m} \xi_{i}^{(m)}(t) \delta_{X_{i}^{(m)}(t)}
$$

defines a $M_{F}(\mathbb{R})$-valued process. From now on we will suppress the dependence of $m$ in both $\xi_{i}^{(m)}$ and $X_{i}^{(m)}$. 
Recall that a collection of processes $\left\{Z^{\alpha}, \alpha \in I\right\}$ with sample paths in $D\left(M_{F}(\mathbb{R})\right)$ is $C$-relatively compact if it is relatively compact and all its weak limits are a.s. continuous. The proof of the next lemma is standard; see, e.g. the proofs for Lemma 3.2 of [18] and Proposition II.4.2 of [12].

Lemma 3.1. $\left\{Z^{(m)}\right\}$ is C-relatively compact.

Proof. We first check the compact containment condition. For any $\epsilon>0$ and $T>0$, choose a compact set $K_{0} \subset D(\mathbb{R})$ such that $\mathbb{P}\left\{X_{1} \in K_{0}^{c}\right\}<\epsilon^{2}$. Let $K:=\left\{x_{t}, x_{t-}: x \in K_{0}, t \leqslant T\right\}$. Then $K$ is compact in $\mathbb{R}$, and

$$
\mathbb{P}\left\{X_{1}(t) \in K^{c}, \exists t \leqslant T\right\} \leqslant \mathbb{P}\left\{X_{1} \in K_{0}^{c}\right\}<\epsilon^{2} .
$$

Define

$$
I_{K}:=\left\{1 \leqslant i \leqslant m: X_{i}(t) \in K^{c}, \exists t \leqslant T\right\}
$$

and put

$$
N_{m}:=\# I_{K}=\sum_{i=1}^{m} 1\left\{X_{i}(t) \in K^{c}, \exists t \leqslant T\right\},
$$

where $\# I_{K}$ denotes the cardinality of the index set $I_{K}$.

Conditioning on $N_{m}$, by the additivity for Feller's branching diffusions, we see that $\sum_{i \in I_{K}} \xi_{i}(t)$ is a Feller's branching diffusion with initial value $N_{m} \bar{z} / m$. Then by Doob's maximal inequality,

$$
\mathbb{P}\left\{\sup _{0 \leqslant t \leqslant T} \sum_{i \in I_{K}} \xi_{i}(t)>\epsilon \mid N_{m}\right\} \leqslant \frac{N_{m} \bar{z}}{m \epsilon} .
$$

Therefore,

$$
\mathbb{P}\left\{\sup _{0 \leqslant t \leqslant T} Z_{t}^{(m)}\left(K^{c}\right)>\epsilon\right\} \leqslant \mathbb{P}\left\{\sup _{0 \leqslant t \leqslant T} \sum_{i \in I_{K}} \xi_{i}(t)>\epsilon\right\} \leqslant \frac{\mathbb{P}\left[N_{m}\right] \bar{z}}{m \epsilon} \leqslant \bar{z} \epsilon .
$$

For any $f \in C_{b}^{2}(\mathbb{R})$, we are going to show that $\left\{\left\langle Z^{(m)}, f\right\rangle\right\}$ is C-relatively compact in $D(\mathbb{R})$. By Itô's formula, we have

$$
\left\langle Z_{t}^{(m)}, f\right\rangle=\sum_{i=1}^{m}\left[\frac{\bar{z}}{m} f\left(x_{i}\right)+\int_{0}^{t} f\left(X_{i}(s)\right) \mathrm{d} \xi_{i}(s)+\int_{0}^{t} \xi_{i}(s) f^{\prime}\left(X_{i}(s)\right) \mathrm{d} X_{i}(s)+\frac{1}{2} \int_{0}^{t} \xi_{i}(s) f^{\prime \prime}\left(X_{i}(s)\right) \mathrm{d} s\right] .
$$

The additivity for $\xi_{i}(t)$ gives that if

$$
\mathbb{P}\left[\sup _{0 \leqslant s \leqslant t} \sum_{i=1}^{m} \xi_{i}(s)\right]<\infty \quad \text { and } \mathbb{P}\left[\sup _{0 \leqslant s \leqslant t} \sum_{i, j=1}^{m} \xi_{i}(s) \xi_{j}(s)\right]<\infty, t>0,
$$

then $\left\{\sum_{i=1}^{m} \int_{0}^{i} \xi_{i}(s) f^{\prime \prime}\left(X_{i}(s)\right) \mathrm{d} s\right\}$ is C-relatively compact following from Arzela-Ascoli theorem and Proposition VI.3.26 of [8].

Note that the quadratic variation

$$
\left\langle\sum_{i=1}^{m} \int_{0} \xi_{i}(s) f^{\prime}\left(X_{i}(s)\right) \mathrm{d} X_{i}(s)\right\rangle_{t}=\sum_{i, j=1}^{m} \int_{0}^{t} \xi_{i}(s) \xi_{j}(s) f^{\prime}\left(X_{i}(s)\right) f^{\prime}\left(X_{j}(s)\right) \mathrm{d}\left\langle X_{i}, X_{j}\right\rangle_{s},
$$

where

$$
\left\langle X_{i}, X_{j}\right\rangle_{s}=s-T_{i j} \wedge s \quad \text { and } \quad T_{i j}:=\inf \left\{s \geqslant 0: X_{i}(s)=X_{j}(s)\right\} .
$$

By Arzela-Ascoli theorem again, $\left\{\left\langle\sum_{i=1}^{m} \int_{0} \xi_{i}(s) f^{\prime}\left(X_{i}(s)\right) \mathrm{d} X_{i}(s)\right\rangle.\right\}$ is C-relatively compact. Theorem VI.4.13 and Proposition VI.3.26 of [8] then imply that the collection of martingales $\left\{\sum_{i=1}^{m} \int_{0} \xi_{i}(s) f^{\prime}\left(X_{i}(s)\right) \mathrm{d} X_{i}(s)\right\}$ is C-relatively compact. 
Similarly, $\left\{\sum_{i=1}^{m} \int_{0} f\left(X_{i}(s)\right) \mathrm{d} \xi_{i}(s)\right\}$ is also C-relatively compact. Moreover,

$$
\frac{1}{m} \sum_{i=1}^{m} f\left(x_{i}\right) \rightarrow\left\langle Z_{0}, f\right\rangle \quad \text { a.s. }
$$

$\left\{\left\langle Z^{(m)}, f\right\rangle\right\}$ is thus C-relatively compact. Consequently, by Theorem II.4.1 of [12] we can conclude that $\left\{Z^{(m)}\right\}$ is C-relatively compact.

Write $Z$ for the weak limit of $\left\{Z^{(m)}\right\}$. The Laplace functional of $Z$ can be obtained from the duality in Theorem 2.1. As a result, its uniqueness is settled.

In the sequel we always write $\left(Y_{1}, \ldots, Y_{2 n}\right)$ for a coalescing Brownian motion starting at $\left(y_{1}, \ldots, y_{2 n}\right)$ with $y_{1} \leqslant \ldots \leqslant y_{2 n}$. Given $\left(a_{i}\right)$ and $t>0$, put

$$
\left.\left.h_{t}(\cdot):=\sum_{j=1}^{n} a_{j} 1\{] Y_{2 j-1}(t), Y_{2 j}(t)\right]\right\}(\cdot) \text {. }
$$

\section{Theorem 3.2.}

(i) Any limit point $Z$ of $\left\{Z^{(m)}\right\}$ in $C\left(M_{F}(\mathbb{R})\right)$ satisfies the following duality relation: given $a_{j}>0, j=1, \ldots, n$, for any $y_{1} \leqslant y_{2} \leqslant \cdots \leqslant y_{2 n}$ and any $t>0$, we have

$$
\mathbb{P}\left[\exp \left\{-\left\langle Z_{t}, h_{0}\right\rangle\right\}\right]=\mathbb{P}\left[\exp \left\{-\left\langle Z_{0}, \frac{2 h_{t}}{2+\gamma t h_{t}}\right\rangle\right\}\right] .
$$

(ii) Any limit point $Z$ of $\left\{Z^{(m)}\right\}$ in $C\left(M_{F}(\mathbb{R})\right)$ has the Markov property.

(iii) The family $\left\{Z^{(m)}\right\}$ has a unique limit point $Z$ in $C\left(M_{F}(\mathbb{R})\right)$.

Proof. We might assume that $\left(Y_{i}\right)$ is independent of $\left(\xi_{i}\right)$. First conditioning on $\left(\xi_{i}(t)\right)$, by Theorem 2.1 we have

$$
\begin{aligned}
& \left.\left.\mathbb{P}\left[\exp \left\{-\sum_{i=1}^{m} \sum_{j=1}^{n} \xi_{i}(t) a_{j} 1\{] y_{2 j-1}, y_{2 j}\right]\right\}\left(X_{i}(t)\right)\right\} \mid\left(\xi_{i}(t)\right)\right] \\
& \left.\left.\quad=\mathbb{P}\left[\exp \left\{-\sum_{i=1}^{m} \sum_{j=1}^{n} \xi_{i}(t) a_{j} 1\{] Y_{2 j-1}(t), Y_{2 j}(t)\right]\right\}\left(x_{i}\right)\right\} \mid\left(\xi_{i}(t)\right)\right] .
\end{aligned}
$$

Now take expectations on both sides of (3.4) and then condition on $\left(x_{i}\right)$ and $\left(Y_{i}(t)\right)$. Since $\xi_{1}(t), \ldots, \xi_{m}(t)$ are independent of each other, and they are independent of $\left(x_{i}\right)$ and $\left(Y_{i}(t)\right)$, it follows from (3.1) that

$$
\begin{aligned}
\mathbb{P}\left[\exp \left\{-\left\langle Z_{t}^{(m)}, h_{0}\right\rangle\right\}\right] & =\mathbb{P}\left[\mathbb{P}\left[\exp \left\{-\sum_{i=1}^{m} \xi_{i}(t) h_{t}\left(x_{i}\right)\right\} \mid\left(x_{i}\right),\left(Y_{i}(t)\right)\right]\right] \\
& =\mathbb{P}\left[\prod_{i=1}^{m} \exp \left\{-\frac{2 \bar{z} h_{t}\left(x_{i}\right)}{m\left\{2+\gamma t h_{t}\left(x_{i}\right)\right\}}\right\}\right] \\
& =\mathbb{P}\left[\mathbb{P}\left[\left\langle\frac{Z_{0}}{\bar{z}}, \exp \left\{-\frac{2 \bar{z} h_{t}}{m\left(2+\gamma t h_{t}\right)}\right\}\right\}^{m} \mid\left(Y_{i}(t)\right)\right]\right] \\
& =\mathbb{P}\left[\left\langle\frac{Z_{0}}{\bar{z}}, \exp \left\{-\frac{2 \bar{z} h_{t}}{m\left(2+\gamma t h_{t}\right)}\right\}\right\}^{m}\right] .
\end{aligned}
$$

Let $m \rightarrow \infty$ in (3.5). Then

$$
\begin{aligned}
\lim _{m \rightarrow \infty} \mathbb{P}\left[\exp \left\{-\left\langle Z_{t}^{(m)}, h_{0}\right\rangle\right\}\right] & =\lim _{m \rightarrow \infty} \mathbb{P}\left[\left(1-\left\langle Z_{0}, \frac{2 h_{t}}{m\left(2+\gamma t h_{t}\right)}\right\rangle\right)^{m}\right] \\
& =\mathbb{P}\left[\exp \left\{-\left\langle Z_{0}, \exp \left\{\frac{2 h_{t}}{2+\gamma t h_{t}}\right\}\right\rangle\right\}\right]
\end{aligned}
$$


Let $Z$ be any limit point of $\left\{Z^{(m)}\right\}$. To prove (3.3) it suffices to show that

$$
\mathbb{P}\left[\exp \left\{-\left\langle Z_{t}, h_{0}\right\rangle\right\}\right]=\lim _{m \rightarrow \infty} \mathbb{P}\left[\exp \left\{-\left\langle Z_{t}^{(m)}, h_{0}\right\rangle\right\}\right] .
$$

To this end we can suppose that $y_{1}<y_{2}<\cdots<y_{2 n}$. Then for small enough $\epsilon>0$, similar to (3.5) we have

$$
\begin{aligned}
& \left.\left.\left.\left.\mathbb{P}\left[\exp \left\{-\sum_{j=1}^{n} a_{j} Z_{t}^{(m)}(] y_{2 j-1}+\epsilon, y_{2 j}-\epsilon\right]\right)\right\}\right]-\mathbb{P}\left[\exp \left\{-\sum_{j=1}^{n} a_{j} Z_{t}^{(m)}(] y_{2 j-1}-\epsilon, y_{2 j}+\epsilon\right]\right)\right\}\right] \\
& \left.\left.\left.\left.\leqslant 1-\mathbb{P}\left[\exp \left\{-\sum_{j=1}^{n} a_{j} Z_{t}^{(m)}(] y_{2 j-1}-\epsilon, y_{2 j-1}+\epsilon\right] \cup\right] y_{2 j}-\epsilon, y_{2 j}+\epsilon\right]\right)\right\}\right] \\
& \quad=1-\mathbb{P}\left[\left\langle\frac{Z_{0}}{\bar{z}}, \exp \left\{-\frac{\left.\left.\left.\left.2 \bar{z} \sum_{j=1}^{n} a_{j} 1\{] Y_{2 j-1}^{\prime}(t), Y_{2 j-1}^{\prime \prime}(t)\right] \cup\right] Y_{2 j}^{\prime}(t), Y_{2 j}^{\prime \prime}(t)\right]\right\}}{\left.\left.\left.\left.m\left(2+\gamma t \sum_{j=1}^{n} a_{j} 1\{] Y_{2 j-1}^{\prime}(t), Y_{2 j-1}^{\prime \prime}(t)\right] \cup\right] Y_{2 j}^{\prime}(t), Y_{2 j}^{\prime \prime}(t)\right]\right\}\right)}\right\}\right\}^{m}\right] \\
& \leqslant 1-\mathbb{P}\left\{\bigcap_{j=1}^{2 n}\left\{Y_{j}^{\prime}(t)=Y_{j}^{\prime \prime}(t)\right\}\right\},
\end{aligned}
$$

where $\left(Y_{1}^{\prime}, Y_{1}^{\prime \prime}, \ldots, Y_{2 n}^{\prime}, Y_{2 n}^{\prime \prime}\right)$ is a coalescing Brownian motion starting at $\left(y_{1}-\epsilon, y_{1}+\epsilon, \ldots, y_{2 n}-\epsilon, y_{2 n}+\epsilon\right)$. Clearly, the right-hand side of (3.7) converges (uniformly in $m$ ) to 0 as $\epsilon \rightarrow 0+$. Therefore, (3.6) follows readily.

By the Markov property for $\left(X_{i}\right)$ and $\left(\xi_{i}\right)$, and arguments similar to (3.5) and (3.6) we can show that, for any $0 \leqslant t_{1}<\cdots<t_{k}<t$ and any nonnegative bounded continuous functions $\left(f_{i}\right)$, we have

$$
\begin{aligned}
& \mathbb{P}\left[\exp \left\{-\sum_{i=1}^{k}\left\langle Z_{t_{i}}, f_{i}\right\rangle-\left\langle Z_{t}, h_{0}\right\rangle\right\}\right] \\
& \quad=\lim _{m \rightarrow \infty} \mathbb{P}\left[\exp \left\{-\sum_{i=1}^{k}\left\langle Z_{t_{i}}^{(m)}, f_{i}\right\rangle-\left\langle Z_{t}^{(m)}, h_{0}\right\rangle\right\}\right] \\
& =\lim _{m \rightarrow \infty} \mathbb{P}\left[\exp \left\{-\sum_{i=1}^{k}\left\langle Z_{t_{i}}^{(m)}, f_{i}\right\rangle\right\} \mathbb{P}\left[\exp \left\{-\left\langle Z_{t}^{(m)}, h_{0}\right\rangle\right\} \mid\left(\xi_{i}(s)\right),\left(X_{i}(s)\right), s \leqslant t_{k}\right]\right] \\
& =\lim _{m \rightarrow \infty} \mathbb{P}\left[\exp \left\{-\sum_{i=1}^{k}\left\langle Z_{t_{i}}^{(m)}, f_{i}\right\rangle\right\} \exp \left\{-\left\langle Z_{t_{k}}^{(m)}, \frac{2 h_{t-t_{k}}}{2+\gamma\left(t-t_{k}\right) h_{t-t_{k}}}\right\rangle\right\}\right] \\
& \quad=\mathbb{P}\left[\exp \left\{-\sum_{i=1}^{k}\left\langle Z_{t_{i}}, f_{i}\right\rangle\right\} \exp \left\{-\left\langle Z_{t_{k}}, \frac{2 h_{t-t_{k}}}{2+\gamma\left(t-t_{k}\right) h_{t-t_{k}}}\right\rangle\right\}\right] .
\end{aligned}
$$

The Markov property for $Z$ follows readily.

Since all the limit points of $\left\{Z^{(m)}\right\}$ have identical marginal distribution because of (3.3), by the Markov property they also have the same joint distribution. We thus establish the uniqueness for $Z$. See Theorem 4.4.2 of [7] and Theorem 3.3 of [18] for similar proofs.

Remark 3.3. The duality (3.2) also gives, for any $0<s<t$,

$$
\mathbb{P}\left[\exp \left\{-\left\langle Z_{t-s}, h_{s}\right\rangle\right\}\right]=\mathbb{P}\left[\exp \left\{-\left\langle Z_{s}, h_{t-s}\right\rangle\right\}\right] .
$$

The moments of $Z$ can be obtained immediately from (3.3).

Proposition 3.4. Given $a_{j}>0, j=1, \ldots, n$, for any $y_{1} \leqslant y_{2} \leqslant \cdots \leqslant y_{2 n}$ and $t>0$, we have

$$
\mathbb{P}\left[\left\langle Z_{t}, h_{0}\right\rangle\right]=\mathbb{P}\left[\left\langle Z_{0}, h_{t}\right\rangle\right]
$$

and

$$
\mathbb{P}\left[\left\langle Z_{t}, h_{0}\right\rangle^{2}\right]=\mathbb{P}\left[\left\langle Z_{0}, h_{t}\right\rangle^{2}\right]+\gamma t \mathbb{P}\left[\left\langle Z_{0}, h_{t}\right\rangle\right] .
$$


Martingale problem is often used to characterize a superprocess. $Z$ is the solution to the martingale problem (see [5]): for any $\psi \in C^{2}(\mathbb{R})$,

$$
M_{t}(\psi):=\left\langle Z_{t}, \psi\right\rangle-\left\langle Z_{0}, \psi\right\rangle-\frac{1}{2} \int_{0}^{t}\left\langle Z_{s}, \psi^{\prime \prime}\right\rangle \mathrm{d} s, \quad t \geqslant 0,
$$

is a continuous martingale relative to $\left(\mathcal{F}_{t}\right)_{t \geqslant 0}$ with quadratic variation process

$$
\langle M(\psi)\rangle_{t}=\gamma \int_{0}^{t}\left\langle Z_{s}, \psi^{2}\right\rangle \mathrm{d} s+\int_{0}^{t} \mathrm{~d} s \int_{\Delta} \psi^{\prime}(x) \psi^{\prime}(y) Z_{s}(\mathrm{~d} x) Z_{s}(\mathrm{~d} y),
$$

where $\Delta=\{(x, x): x \in \mathbb{R}\}$.

But a remarkable feature of such a martingale problem is that its solution is not unique. For example, let $\xi_{1}$ and $\xi_{2}$ be two independent branching diffusions each with initial value 1. Let $B_{1}$ and $B_{2}$ be two independent Brownian motions. Assume that $\left(\xi_{1}, \xi_{2}\right)$ and $\left(B_{1}, B_{2}\right)$ are independent. Then $Z_{t}^{\prime}:=\xi_{1}(t) \delta_{B_{1}(t)}+\xi_{2}(t) \delta_{B_{1}(t)}$ is another solution to this martingale problem; also see [18] for a similar counter example.

Uniqueness of the solution to a martingale problem is often established by finding an appropriate dual process via the method of martingale duality; see Section 1.6 of [6] for an introduction of such an approach. Notice that the duality (3.3) is not a consequence of the martingale duality corresponding to the above mentioned martingale problem. Not surprisingly, it cannot guarantee the uniqueness of the solution.

\section{Some properties}

Our first result in this section is a straight forward consequence of Theorem 3.2.

Proposition 4.1. For any $y_{1} \leqslant y_{2} \leqslant \cdots \leqslant y_{2 n}$ and $t>0$, we have

$$
\left.\left.\left.\left.\mathbb{P}\left[\exp \left\{-\lambda \sum_{j=1}^{n} Z_{t}(] y_{2 j-1}, y_{2 j}\right]\right)\right\}\right]=\mathbb{P}\left[\exp \left\{-\frac{2 \lambda}{2+\lambda \gamma t} \sum_{j=1}^{n} Z_{0}(] Y_{2 j-1}(t), Y_{2 j}(t)\right]\right)\right\}\right], \quad \lambda>0 .
$$

Proof. Observe that the function $\left.\left.\sum_{j=1}^{n} 1\{] Y_{2 j-1}(t), Y_{2 j}(t)\right]\right\}(\cdot)$ takes values either 0 or 1 , then (4.1) follows readily from (3.3).

Proposition 4.1 allows us to carry out some explicit computation on $Z$. We are going to first study the probability that $Z_{t}$ does not charge on an arbitrary finite interval.

Throughout this section, for any $x, y, a$ and $b$, we write

$$
\tilde{x}:=\frac{x-y}{\sqrt{2}}, \quad \tilde{y}:=\frac{x+y}{\sqrt{2}}, \quad \tilde{a}:=\frac{a+b}{\sqrt{2}} \quad \text { and } \quad \tilde{b}:=\frac{b-a}{\sqrt{2}} .
$$

Proposition 4.2. Given $a<b$ and $t>0$, we have

$$
\begin{aligned}
\left.\left.\mathbb{P}\left\{Z_{t}(] a, b\right]\right)=0\right\}= & \frac{1}{2 \pi t} \int_{-\infty}^{\infty} \mathrm{d} x \int_{0}^{\infty} \mathrm{d} y \exp \left\{-\frac{\left.\left.2 Z_{0}(] \tilde{x}, \tilde{y}\right]\right)}{t \gamma}-\frac{(x-\tilde{a})^{2}}{2 t}\right\} \\
& \times\left(\exp \left\{-\frac{(y-\tilde{b})^{2}}{2 t}\right\}-\exp \left\{-\frac{(y+\tilde{b})^{2}}{2 t}\right\}\right)+\frac{2}{\sqrt{2 \pi t}} \int_{\tilde{b}}^{\infty} \mathrm{d} x \exp \left\{-\frac{x^{2}}{2 t}\right\} .
\end{aligned}
$$

Proof. Letting $\lambda \rightarrow \infty$ in (4.1) we have 


$$
\begin{aligned}
\left.\left.\mathbb{P}\left\{Z_{t}(] a, b\right]\right)=0\right\} & \left.\left.=\mathbb{P}\left[\exp \left\{-\frac{2}{\gamma t} Z_{0}(] Y_{1}(t), Y_{2}(t)\right]\right)\right\}\right] \\
& \left.\left.=\mathbb{P}\left[\exp \left\{-\frac{2}{\gamma t} Z_{0}(] Y_{1}(t), Y_{2}(t)\right]\right)\right\} ; Y_{1}(t) \neq Y_{2}(t)\right]+\mathbb{P}\left\{Y_{1}(t)=Y_{2}(t)\right\},
\end{aligned}
$$

where $\left(Y_{1}, Y_{2}\right)$ is a coalescing Brownian motion starting at $(a, b)$.

To find the distribution of $\left(Y_{1}, Y_{2}\right)$, one could rotate the coordinate system anti-clockwise by $\pi / 4$. Under the new coordinate system $\left(Y_{1}, Y_{2}\right)$ becomes a process $\left(Y_{1}^{\prime}, Y_{2}^{\prime}\right)$ such that $Y_{1}^{\prime}$ is a Brownian motion starting at $\tilde{a}, Y_{2}^{\prime}$ is a Brownian motion starting at $\tilde{b}$ and stopped at 0 , and $Y_{1}^{\prime}$ and $Y_{2}^{\prime}$ are independent. Since $Y_{1}=\left(Y_{1}^{\prime}-Y_{2}^{\prime}\right) / \sqrt{2}, Y_{2}=$ $\left(Y_{1}^{\prime}+Y_{2}^{\prime}\right) / \sqrt{2}$ and $Y_{1}(t)=Y_{2}(t)$ iff $Y_{2}^{\prime}(t)=0$, then (4.3) just follows from the reflection principle for Brownian motion.

Write $S_{t}$ for the support of $Z_{t}$. Intuitively, starting with particles with total initial mass $Z_{0}(\mathbb{R})$, as soon as $t>0$ the particles near $-\infty$ and $\infty$ will die out due to branching. $Z_{t}$ is then expected to be supported by a finite set because of coalescence. The next two results concern the cardinality of $S_{t}$.

Proposition 4.3. Given $a<b$ and $t>0$, we have

$$
\left.\left.\mathbb{P}\left[\# S_{t} \cap\right] a, b\right]\right]=\frac{b-a}{\sqrt{\pi t}}-\frac{1}{\sqrt{2} \pi t^{2}} \int_{a}^{b} \mathrm{~d} z \int_{-\infty}^{\infty} \mathrm{d} x \int_{0}^{\infty} \mathrm{d} y y \exp \left\{-\frac{\left.\left.2 Z_{0}(] \tilde{x}, \tilde{y}\right]\right)}{t \gamma}-\frac{(x-\sqrt{2} z)^{2}+y^{2}}{2 t}\right\} .
$$

Proof. It is easy to see from (4.3) that for any $z \in \mathbb{R}$,

$$
\mathbb{P}\left\{Z_{t}(\mathrm{~d} z) \neq 0\right\}=\frac{\mathrm{d} z}{\sqrt{\pi t}}-\frac{\mathrm{d} z}{\sqrt{2} \pi t^{2}} \int_{-\infty}^{\infty} \mathrm{d} x \int_{0}^{\infty} \mathrm{d} y y \exp \left\{-\frac{\left.\left.2 Z_{0}(] \tilde{x}, \tilde{y}\right]\right)}{t \gamma}-\frac{(x-\sqrt{2} z)^{2}+y^{2}}{2 t}\right\} .
$$

Then (4.4) is obtained by taking integrals on both sides of (4.5) from $a$ to $b$.

Proposition 4.4. With probability $1, \# S_{t}<\infty, \forall t>0$.

Proof. Given $s>0$, we first claim that $\mathbb{P}\left[\# S_{s}\right]<\infty$ if $Z_{0}$ has a bounded support. Suppose that $\left.\left.Z_{0}(]-b, b\right]\right)=1$ for some $b>0$. Then by (4.5),

$$
\begin{aligned}
\mathbb{P}\left[\# S_{s}\right] & =\int_{-\infty}^{\infty} \mathbb{P}\left\{Z_{s}(\mathrm{~d} z) \neq 0\right\} \\
& =\frac{1}{\sqrt{2} \pi s^{2}} \int_{-\infty}^{\infty} \mathrm{d} z \int_{-\infty}^{\infty} \mathrm{d} x \int_{0}^{\infty} \mathrm{d} y y\left(1-\exp \left\{-\frac{\left.\left.2 Z_{0}(] \tilde{x}, \tilde{y}\right]\right)}{s \gamma}\right\}\right) \exp \left\{-\frac{(x-\sqrt{2} z)^{2}+y^{2}}{2 s}\right\} \\
& =\frac{1}{\sqrt{2 \pi s}} \int_{-\infty}^{\infty} \mathrm{d} x \int_{0}^{\infty} \mathrm{d} y y\left(1-\exp \left\{-\frac{\left.\left.2 Z_{0}(] \tilde{x}, \tilde{y}\right]\right)}{s \gamma}\right\}\right) \exp \left\{-\frac{y^{2}}{2 s}\right\} \\
& \leqslant \frac{1}{\sqrt{2 \pi s}} \int_{0}^{\infty} \mathrm{d} y \int_{-y-\sqrt{2} b}^{y+\sqrt{2} b} \mathrm{~d} x y \exp \left\{-\frac{y^{2}}{2 s}\right\} \\
& <\infty
\end{aligned}
$$

Our claim is proved. 
Now given any integer $j$, let $\eta_{j}(s)$ be the Feller's branching diffusion with initial value $\left.\left.\eta_{j}(0):=Z_{0}(] j, j+1\right]\right)$. Since

$$
\sum_{j=-\infty}^{\infty} \mathbb{P}\left\{\eta_{j}(s) \neq 0\right\}=\sum_{j=-\infty}^{\infty}\left(1-\exp \left\{-\frac{2 \eta_{j}(0)}{\gamma s}\right\}\right) \leqslant \sum_{j=-\infty}^{\infty} \frac{2 \eta_{j}(0)}{\gamma s}=\frac{2 \bar{z}}{\gamma s},
$$

by Borel-Cantelli lemma we have that, with probability $1, \eta_{j}(s) \neq 0$ for only finitely many values of $j$.

Therefore, for any $t>0$, with probability $1, Z_{t / 2}$ must have a bounded support. The Markov property for $Z$, together with the claim from the first part of the proof, implies that $\# S_{t}<\infty$ a.s.

Finally, by the Markov property for $Z$ we conclude that $\mathbb{P}\left\{\# S_{t}<\infty, \forall t>0\right\}=1$.

By Proposition 4.4, as soon as $t>0, S_{t}$ becomes a finite set. For any $z \in S_{t}$, we associate it with a particle located at $z$ with mass $Z_{t}(\{z\})$. We can thus identify $Z_{t}$ interchangeably with a collection of spatially distributed particles with masses. As time goes on, the total number of particles decreases either because two "alive" particles coalesce into one particle, or because each particle disappears due to its branching.

Since $\# S_{t}<\infty$, a small neighborhood of $z$ contains at most one particle in $Z_{t}$. When there is such a particle, we want to find the distribution of its mass. Formally, we are looking for an expression of

$$
\mathbb{P}\left[\exp \left\{-\lambda Z_{t}(\{z\})\right\} ; Z_{t}(\{z\})>0\right] .
$$

Until the end of this section we put

$$
\left.\left.q_{t}(\cdot):=1\{] Y_{1}(t), Y_{2}(t)\right]\right\}(\cdot), \quad t \geqslant 0,
$$

for the coalescing Brownian motion $\left(Y_{1}, Y_{2}\right)$ starting at $(a, b)$.

Proposition 4.5. For any $z \in \mathbb{R}$ and $t>0$, we have

$$
\begin{aligned}
\mathbb{P}\left[\exp \left\{-\lambda Z_{t}(\mathrm{~d} z)\right\} ; Z_{t}(\mathrm{~d} z)>0\right]= & \frac{\mathrm{d} z}{\sqrt{2} \pi t^{2}} \int_{-\infty}^{\infty} \mathrm{d} x \int_{0}^{\infty} \mathrm{d} y y\left(\exp \left\{-\frac{\left.\left.2 \lambda Z_{0}(] \tilde{x}, \tilde{y}\right]\right)}{2+\lambda \gamma t}\right\}-\exp \left\{-\frac{\left.\left.2 Z_{0}(] \tilde{x}, \tilde{y}\right]\right)}{t \gamma}\right\}\right) \\
& \times \exp \left\{-\frac{(x-\sqrt{2} z)^{2}+y^{2}}{2 t}\right\} .
\end{aligned}
$$

Proof. We fix $\left(\xi_{i}(t)\right)$ first. Apply Theorem 2.1 to random variable

$$
\exp \left\{-\lambda \sum_{i=1}^{m} \xi_{i}(t) q_{0}\left(X_{i}(t)\right)\right\} 1\left\{\sum_{i=1}^{m} \xi_{i}(t) q_{0}\left(X_{i}(t)\right)>0\right\} .
$$

Then condition on $\left(Y_{1}(t), Y_{2}(t)\right)$ and take an expectation with respect to $\left(\xi_{i}(t)\right)$. Similar to the proofs for Theorem 3.2 and for Proposition 4.1 we have that

$$
\begin{aligned}
\mathbb{P} & {\left[\exp \left\{-\lambda \sum_{i=1}^{m} \xi_{i}(t) q_{0}\left(X_{i}(t)\right)\right\} ; \sum_{i=1}^{m} \xi_{i}(t) q_{0}\left(X_{i}(t)\right)>0\right] } \\
& =\mathbb{P}\left[\exp \left\{-\lambda \sum_{i=1}^{m} \xi_{i}(t) q_{t}\left(x_{i}\right)\right\} ; \sum_{i=1}^{m} \xi_{i}(t) q_{t}\left(x_{i}\right)>0\right] \\
& =\mathbb{P}\left[\exp \left\{-\lambda \sum_{i=1}^{m} \xi_{i}(t) q_{t}\left(x_{i}\right)\right\}\right]-\mathbb{P}\left[\sum_{i=1}^{m} \xi_{i}(t) q_{t}\left(x_{i}\right)=0\right] \\
& =\mathbb{P}\left[\exp \left\{-\frac{2 \lambda\left\langle Z_{0}^{(m)}, q_{t}\right\rangle}{2+\lambda \gamma t}\right\}\right]-\mathbb{P}\left[\exp \left\{-\frac{2\left\langle Z_{0}^{(m)}, q_{t}\right\rangle}{\gamma t}\right\}\right] .
\end{aligned}
$$

Recall that $\tilde{x}, \tilde{y}, \tilde{a}$ and $\tilde{b}$ have been defined in (4.2). Therefore, 


$$
\begin{aligned}
\left.\left.\left.\left.\mathbb{P}\left[\exp \left\{-\lambda Z_{t}(] a, b\right]\right)\right\} ; Z_{t}(] a, b\right]\right)>0\right] \\
=\mathbb{P}\left[\exp \left\{-\frac{2 \lambda\left\langle Z_{0}, q_{t}\right\rangle}{2+\lambda \gamma t}\right\}\right]-\mathbb{P}\left[\exp \left\{-\frac{2\left\langle Z_{0}, q_{t}\right\rangle}{\gamma t}\right\}\right] \\
=\frac{1}{2 \pi t} \int_{-\infty}^{\infty} \mathrm{d} x \int_{0}^{\infty} \mathrm{d} y\left(\exp \left\{-\frac{\left.\left.2 \lambda Z_{0}(] \tilde{x}, \tilde{y}\right]\right)}{2+\lambda \gamma t}\right\}-\exp \left\{-\frac{\left.\left.2 Z_{0}(] \tilde{x}, \tilde{y}\right]\right)}{t \gamma}\right\}\right) \\
\quad \times \exp \left\{-\frac{(x-\tilde{a})^{2}}{2 t}\right\}\left(\exp \left\{-\frac{(y-\tilde{b})^{2}}{2 t}\right\}-\exp \left\{-\frac{(y+\tilde{b})^{2}}{2 t}\right\}\right) .
\end{aligned}
$$

So, (4.6) is obtained by letting $b \rightarrow a+$.

At a fixed time $t>0$, with a positive probability there can be only one particle (with a positive mass) left. When this happens, we are interested in the joint distribution of the mass and the location of that particle. More precisely, we want to find an expression for

$$
\mathbb{P}\left[\exp \left\{-\lambda Z_{t}(\mathbb{R})\right\} ; Z_{t}(\mathbb{R}) \neq 0, S_{t} \subset \mathrm{d} z\right] .
$$

Proposition 4.6. For any $z \in \mathbb{R}$ and $t>0$, we have

$$
\begin{aligned}
\mathbb{P} & \left.\exp \left\{-\lambda Z_{t}(\mathbb{R})\right\} ; Z_{t}(\mathbb{R}) \neq 0, S_{t} \subset \mathrm{d} z\right] \\
= & \frac{\mathrm{d} z}{\sqrt{2} \pi t^{2}} \int_{-\infty}^{\infty} \mathrm{d} x \int_{0}^{\infty} \mathrm{d} y y \exp \left\{-\frac{\left.\left.2 \lambda Z_{0}(] \tilde{x}, \tilde{y}\right]\right)}{2+\lambda \gamma t}-\frac{\left.\left.2 Z_{0}(] \tilde{x}, \tilde{y}\right]^{c}\right)}{\gamma t}-\frac{(x-\sqrt{2} z)^{2}+y^{2}}{2 t}\right\} \\
& -\frac{\mathrm{d} z}{\sqrt{\pi t}} \exp \left\{-\frac{2 \bar{z}}{\gamma t}\right\} .
\end{aligned}
$$

Proof. For $x_{i}:=X_{i}(0)$ we put

$$
\left.\left.B:=\left\{\sum_{i=1}^{m} \xi_{i}(t) 1\{] Y_{1}(t), Y_{2}(t)\right]^{c}\right\}\left(x_{i}\right)=0\right\}=\left\{\sum_{i=1}^{m} \xi_{i}(t)\left(1-q_{t}\left(x_{i}\right)\right)=0\right\} .
$$

It follows from Theorem 2.1 that

$$
\begin{aligned}
\left.\left.\left.\left.\mathbb{P}\left[\exp \left\{-\lambda \sum_{i=1}^{m} \xi_{i}(t)\right\} ; \sum_{i=1}^{m} \xi_{i}(t) 1\{] a, b\right]\right\}\left(X_{i}(t)\right)>0, \sum_{i=1}^{m} \xi_{i}(t) 1\{] a, b\right]^{c}\right\}\left(X_{i}(t)\right)=0\right] \\
=\mathbb{P}\left[\exp \left\{-\lambda \sum_{i=1}^{m} \xi_{i}(t)\right\} ; \sum_{i=1}^{m} \xi_{i}(t) q_{t}\left(x_{i}\right)>0, B\right] \\
=\mathbb{P}\left[\exp \left\{-\lambda \sum_{i=1}^{m} \xi_{i}(t) q_{t}\left(x_{i}\right)\right\} ; \sum_{i=1}^{m} \xi_{i}(t) q_{t}\left(x_{i}\right)>0, B\right] \\
=\mathbb{P}\left[\exp \left\{-\lambda \sum_{i=1}^{m} \xi_{i}(t) q_{t}\left(x_{i}\right)\right\} ; B\right]-\mathbb{P}\left[\sum_{i=1}^{m} \xi_{i}(t) q_{t}\left(x_{i}\right)=0 ; B\right] \\
=\mathbb{P}\left[\exp \left\{-\frac{2 \lambda \bar{z} \sum_{i=1}^{m} q_{t}\left(x_{i}\right)}{m(2+\lambda \gamma t)}\right\} \exp \left\{-\frac{2 \bar{z} \sum_{i=1}^{m}\left(1-q_{t}\left(x_{i}\right)\right)}{m \gamma t}\right\}\right]-\mathbb{P}\left\{\sum_{i=1}^{m} \xi_{i}(t)=0\right\}
\end{aligned}
$$

where in obtaining the last equation we have used the fact that, given $\left(Y_{1}(t), Y_{2}(t)\right)$ and $\left(x_{i}\right)$, random variable $\exp \left\{-\lambda \sum_{i=1}^{m} \xi_{i}(t) q_{t}\left(x_{i}\right)\right\}$ and event $\left\{\sum_{i=1}^{m} \xi_{i}(t)\left(1-q_{t}\left(x_{i}\right)\right)=0\right\}$ are independent.

Now letting $m \rightarrow \infty$ in (4.10) we have 


$$
\begin{aligned}
\mathbb{P}\left[\exp \left\{-\lambda Z_{t}(\mathbb{R})\right\} ; Z_{t}(\mathbb{R}) \neq 0, S_{t} \subset(a, b)\right] \\
=\mathbb{P}\left[\exp \left\{-\frac{2 \lambda\left\langle Z_{0}, q_{t}\right\rangle}{2+\lambda \gamma t}-\frac{2\left\langle Z_{0}, 1-q_{t}\right\rangle}{\gamma t}\right\}\right]-\exp \left\{-\frac{2 \bar{z}}{\gamma t}\right\} \\
=\frac{1}{2 \pi t} \int_{-\infty}^{\infty} \mathrm{d} x \int_{0}^{\infty} \mathrm{d} y \exp \left\{-\frac{\left.\left.2 \lambda Z_{0}(] \tilde{x}, \tilde{y}\right]\right)}{2+\lambda \gamma t}-\frac{\left.\left.2 Z_{0}(] \tilde{x}, \tilde{y}\right]^{c}\right)}{\gamma t}\right\} \exp \left\{-\frac{(x-\tilde{a})^{2}}{2 t}\right\} \\
\quad \times\left(\exp \left\{-\frac{(y-\tilde{b})^{2}}{2 t}\right\}-\exp \left\{-\frac{(y+\tilde{b})^{2}}{2 t}\right\}\right) \\
+\frac{2 \bar{z}}{\sqrt{2 \pi t}} \exp \left\{-\frac{2}{\gamma t}\right\} \int_{\tilde{b}}^{\infty} \mathrm{d} x \exp \left\{-\frac{x^{2}}{2 t}\right\}-\exp \left\{-\frac{2 \bar{z}}{\gamma t}\right\} .
\end{aligned}
$$

Finally, (4.9) is obtained by letting $b \rightarrow a+$ in (4.11).

Remark 4.7. Let $\lambda=0$ in (4.11). We then obtain a result on the range of $S_{t}$.

The total number of particles in $Z$ will decrease one by one. Put

$$
\tau:=\inf \left\{s \geqslant 0: \# S_{s}=1\right\} .
$$

Then $\tau<\infty$ is the first time when there is exactly one particle left. The distribution of $\tau$ is given in the following proposition.

\section{Proposition 4.8.}

$$
\begin{aligned}
\mathbb{P}\{\tau \leqslant t\}= & \int_{-\infty}^{\infty} \mathrm{d} z\left(\frac{1}{\sqrt{2} \pi t^{2}} \int_{-\infty}^{\infty} \mathrm{d} x \int_{0}^{\infty} \mathrm{d} y y \exp \left\{-\frac{\left.\left.2 Z_{0}(] \tilde{x}, \tilde{y}\right]^{c}\right)}{\gamma t}-\frac{(x-\sqrt{2} z)^{2}+y^{2}}{2 t}\right\}\right. \\
& \left.-\frac{1}{\sqrt{\pi t}} \exp \left\{-\frac{2 \bar{z}}{\gamma t}\right\}\right)+\exp \left\{-\frac{2 \bar{z}}{\gamma t}\right\} .
\end{aligned}
$$

Proof. Observe that

$$
\mathbb{P}\{\tau \leqslant t\}=\int_{-\infty}^{\infty} \mathbb{P}\left\{Z_{t}(\mathbb{R}) \neq 0, S_{t} \subset \mathrm{d} z\right\}+\mathbb{P}\left\{Z_{t}(\mathbb{R})=0\right\},
$$

then (4.12) follows from Propositions 4.6 and 4.1.

Let

$$
T:=\inf \left\{t \geqslant 0: Z_{t}(\mathbb{R})=0\right\} .
$$

$T$ is the time when all the particles disappear. Its distribution can be easily found from Proposition 4.1.

$$
\mathbb{P}\{T \leqslant t\}=\mathbb{P}\left\{Z_{t}(\mathbb{R})=0\right\}=\exp \left\{-\frac{2 \bar{z}}{\gamma t}\right\} .
$$

Note that super Brownian motion has the same extinction time distribution.

Let $F$ denote the location of the last particle immediately before extinction, i.e. $\{F\}=S_{T-}$. We could recover the explicit distribution for $F$.

Proposition 4.9. $F$ has the same distribution as $X_{T}$, where $X$ is a Brownian motion with initial distribution $Z_{0} / \bar{z}$, and $X$ and $T$ are independent. 
Proof. We first assume that

$$
Z_{0}=\sum_{i=1}^{m} c_{i} \delta_{x_{i}}, \quad c_{i}>0, i=1, \ldots, m .
$$

Then by Theorem 3.2,

$$
Z_{t}=\sum_{i=1}^{m} \xi_{i}(t) \delta_{X_{i}(t)}, \quad t \geqslant 0
$$

where $\left(\xi_{i}\right)$ is a collection of independent Feller's branching diffusions with initial values $\left(c_{i}\right),\left(X_{i}\right)$ is a coalescing Brownian motion starting at $\left(x_{i}\right)$, and $\left(\xi_{i}\right)$ and $\left(X_{i}\right)$ are independent.

Write

$$
T_{i}:=\inf \left\{t \geqslant 0: \xi_{i}(t)=0\right\}, \quad i=1, \ldots, m .
$$

Then

$$
T=\max _{1 \leqslant i \leqslant m} T_{i}
$$

Therefore,

$$
F=\sum_{i=1}^{m} X_{i}\left(T_{i}-\right) 1\left\{T=T_{i}\right\}=\sum_{i=1}^{m} X_{i}(T) 1\left\{T=T_{i}\right\} .
$$

Our first observation is that

$$
\mathbb{P}\left\{T_{i} \leqslant t\right\}=\mathbb{P}\left\{\xi_{i}(t)=0\right\}=\exp \left\{-\frac{2 c_{i}}{\gamma t}\right\} .
$$

Put $c:=\sum_{i=1}^{m} c_{i}$. Then $\mathbb{P}\left\{T=T_{i}\right\}=c_{i} / c$, and $F=X_{i}(T)$ with probability $c_{i} / c$. Our second observation is that conditional on $\left\{T=T_{i}\right\}$, the distribution for $T$ is the same as its unconditional distribution. So, $F$ has the same distribution as the random variable obtained by running a Brownian motion $X$ with initial distribution $\mathbb{P}\{X(0)=$ $\left.x_{i}\right\}=c_{i} / c, i=1, \ldots, m$, and stopping it independently at time $T$. As a result, $F$ has the desired distribution.

By conditioning on $Z_{\epsilon}$ and letting $\epsilon \rightarrow 0+$, the conclusion in the proposition also follows for any general initial measure $Z_{0}$.

Remark 4.10. This near extinction behavior is the same as that for super Brownian motion (see Theorem 1 of [17]), which appears to be coincidental.

\section{Connections with the Arratia flow}

Arratia flow is a stochastic flow which describes the evolution of a continuous family of coalescing Brownian motions on $\mathbb{R}$. We refer to [1] for a detailed account and [2] for a survey on stochastic flows. We also refer to [10] for more recent work on stochastic flows. By definition, the Arratia flow is a collection $\{\phi(s, t, x): 0 \leqslant s \leqslant t, x \in \mathbb{R}\}$ of real-valued random variables such that

- the random map $(s, t, x) \mapsto \phi(s, t, x)$ is jointly measurable,

- for each $s$ and $x$, the map $t \mapsto \phi(s, t, x), t \geqslant s$, is continuous,

- for each $s$ and $t$ with $s \leqslant t$, the map $x \mapsto \phi(s, t, x)$ is non-decreasing and right-continuous,

- for $s \leqslant t \leqslant u, \phi(t, u, \cdot) \circ \phi(s, t, \cdot)=\phi(s, u, \cdot)$,

- for $u>0,(s, t, x) \mapsto \phi(s+u, t+u, x)$ has the same distribution as $\phi$,

- for $x_{1}<\cdots<x_{m}$ the process $\left(\phi\left(0, t, x_{1}\right), \ldots, \phi\left(0, t, x_{m}\right)\right)_{t \geqslant 0}$ has the same distribution as a coalescing Brownian motion starting at $\left(x_{1}, \ldots, x_{m}\right)$. 
Fix $t>0$, it is known that $\{\phi(0, t, x): x \in \mathbb{R}\}$, the image of $\mathbb{R}$ under the map $\phi(0, t, \cdot)$, is a discrete set (see Theorem 12 of Chapter Three in [1]). In this section, let $\cdots<x_{-1}(t)<x_{0}(t)<x_{1}(t)<\cdots$ be a sequence of random variables such that

$$
\{\phi(0, t, x): x \in \mathbb{R}\}=\left\{x_{i}(t): i=\ldots,-1,0,1, \ldots\right\} .
$$

Since Brownian motion has continuous sample paths, the Arratia flow is order-preserving; i.e. $\phi\left(0, t, x_{1}\right) \leqslant \cdots \leqslant$ $\phi\left(0, t, x_{m}\right)$ whenever $x_{1} \leqslant \cdots \leqslant x_{m}$. Set

$$
\Pi_{i}(t):=\sup \left\{x: \phi(0, t, x)=x_{i}(t)\right\} .
$$

Write $\phi^{-1}(0, t, x)$ for the pre-image of $x$ under map $\phi(0, t, \cdot)$ Then $\left(\Pi_{i}(t)\right)$ determines a partition on $\mathbb{R}$ such that $\phi^{-1}\left(0, t, x_{i}(t)\right)=\left[\Pi_{i-1}(t), \Pi_{i}(t)[\right.$.

The Arratia flow is closely associated to the process $Z$ studied in the previous sections. We first want to show that the Laplace functional of $Z_{t}$ for a continuous test function can be expressed in terms of $\left(x_{i}(t)\right)$ and $\left(\Pi_{i}(t)\right)$.

Proposition 5.1. Given any nonnegative bounded continuous function $f$, we have for $t>0$,

$$
\mathbb{P}\left[\exp \left\{-\left\langle Z_{t}, f\right\rangle\right\}\right]=\mathbb{P}\left[\exp \left\{-\left\langle Z_{0}, \frac{2 h_{t}^{f}}{2+\gamma t h_{t}^{f}}\right)\right\}\right]
$$

where $\left.\left.h_{t}^{f}(\cdot):=\sum_{i=-\infty}^{\infty} f\left(\Pi_{i}(t)\right) 1\{] x_{i}(t), x_{i+1}(t)\right]\right\}(\cdot)$.

Proof. For $y_{j}=j / 2^{n}$, Theorem 3.2 yields

$$
\begin{aligned}
& \left.\left.\mathbb{P}\left[\exp \left\{-\sum_{j=-n 2^{n}}^{n 2^{n}} f\left(y_{j}\right) Z_{t}(] y_{j-1}, y_{j}\right]\right)\right\}\right] \\
& \quad=\mathbb{P}\left[\exp \left\{-\left\langle Z_{0}, \frac{\left.\left.2 \sum_{j=-n 2^{n}}^{n 2^{n}} f\left(y_{j}\right) 1\{] \phi\left(0, t, y_{j-1}\right), \phi\left(0, t, y_{j}\right)\right]\right\}}{\left.\left.2+\gamma t \sum_{j=-n 2^{n}}^{n 2^{n}} f\left(y_{j}\right) 1\{] \phi\left(0, t, y_{j-1}\right), \phi\left(0, t, y_{j}\right)\right]\right\}}\right\rangle\right\} .\right.
\end{aligned}
$$

Observe that $\phi\left(0, t, y_{j-1}\right) \neq \phi\left(0, t, y_{j}\right)$ if and only if $y_{j-1}<\Pi_{k}(t) \leqslant y_{j}$ for some $k$. Letting $n \rightarrow \infty$ in (5.3), (5.2) follows from the continuity for $f$ and the finiteness for $Z_{0}$.

We then consider the support $S_{t}$ for $Z_{t}$. Since $S_{t}$ is a discrete set, we can identify it with a measure-valued process by placing a unit mass on each point of $S_{t}$. For any $y_{1} \leqslant y_{2} \leqslant \cdots \leqslant y_{2 n}$, by Proposition 4.1 ,

$$
\left.\left.\left.\mathbb{P}\left\{Z_{t}\left(\bigcup_{j=1}^{n}\right] y_{2 j-1}, y_{2 j}\right]\right)=0\right\}=\mathbb{P}\left[\exp \left\{-\frac{2}{\gamma t} Z_{0}\left(\bigcup_{j=1}^{n}\right] Y_{2 j-1}(t), Y_{2 j}(t)\right]\right)\right\} .
$$

We thus get the following characterization of the avoidance function for $S_{t}$.

$$
\left.\left.\left.\left.\mathbb{P}\left\{S_{t} \cap \bigcup_{j=1}^{n}\right] y_{2 j-1}, y_{2 j}\right]=\emptyset\right\}=\mathbb{P}\left[\exp \left\{-\frac{2}{\gamma t} Z_{0}\left(\bigcup_{j=1}^{n}\right] Y_{2 j-1}(t), Y_{2 j}(t)\right]\right)\right\}\right] .
$$

Consequently the distribution of $S_{t}$ is uniquely determined by (5.5); see Theorem 3.3 of [9].

(5.5) suggests a connection between $S_{t}$ and the Arratia flow. Let $I_{t}(\mathrm{~d} y)$ be a random measure on $\mathbb{R}$ such that

$$
\left.\left.\left.\left.I_{t}\left(\bigcup_{j=1}^{n}\right] y_{2 j-1}, y_{2 j}\right]\right)=\frac{2}{\gamma t} \sum_{j=1}^{n} Z_{0}(] \phi\left(0, t, y_{2 j-1}\right), \phi\left(0, t, y_{2 j}\right)\right]\right), \quad y_{1} \leqslant y_{2} \leqslant \cdots \leqslant y_{2 n} .
$$


Proposition 5.2. Given $t>0, S_{t}$ can be identified with a Cox process with finite random intensity measure $I_{t}$.

(5.4) also leads to a result on the occupation time for $Z$. For any Borel set $B$ in $\mathbb{R}$,

$$
\int_{0}^{t} \mathrm{~d} s \mathbb{P}\left\{Z_{S}(B)=0\right\}=\int_{0}^{t} \mathrm{~d} s \mathbb{P}\left[\exp \left\{-I_{S}(B)\right\}\right] .
$$

A particle representation for $Z_{t}$ is available by using the image of the Arratia flow as a skeleton. Given $\left(x_{i}(t)\right)$ as in (5.1), let $\left(\eta_{i}(t)\right)_{i=-\infty}^{\infty}$ be independent nonnegative random variables such that

$$
\mathbb{P}\left[\exp \left\{-\lambda \eta_{i}(t)\right\} \mid\left(x_{i}(t)\right)\right]=\exp \left\{-\frac{2 \lambda Z_{0}\left(\phi^{-1}\left(0, t, x_{i}(t)\right)\right)}{2+\lambda \gamma t}\right\} .
$$

Proposition 5.3. Given $t>0$, we have

$$
Z_{t} \stackrel{D}{=} \sum_{i=-\infty}^{\infty} \eta_{i}(t) \delta_{x_{i}(t)}
$$

Proof. Define

$$
Z_{t}^{(m)}:=\sum_{i=-m 2^{m}}^{m 2^{m}} \eta_{i}^{(m)}(t) \delta_{\phi\left(0, t, i / 2^{m}\right)},
$$

where $\left(\eta_{i}^{(m)}\right)_{i=-m 2^{m}}^{m 2^{m}}$ is a sequence of independent Feller's branching diffusions with initial values

$$
\left(Z _ { 0 } \left(\left[\frac{i-1}{2^{m}}, \frac{i}{2^{m}}[)\right)_{i=-m 2^{m}}^{m 2^{m}},\right.\right.
$$

and in addition, $\left(\eta_{i}^{(m)}\right)$ is independent of $\left\{x_{i}(t)\right\}$.

For any $a_{j} \geqslant 0, j=1, \ldots, n$, and $y_{1} \leqslant \cdots \leqslant y_{2 n}$, by the same argument as in the proof for Theorem 3.2, we have

$$
\begin{aligned}
\lim _{m \rightarrow \infty} \mathbb{P}\left[\exp \left\{-\left\langle Z_{t}^{(m)}, h_{0}\right\rangle\right\}\right] & =\lim _{m \rightarrow \infty} \mathbb{P}\left[\prod_{i=-m 2^{m}}^{m 2^{m}} \exp \left\{-\frac{2 Z_{0}\left(\left[(i-1) / 2^{m}, i / 2^{m}[) h_{t}\left(i / 2^{m}\right)\right.\right.}{2+\gamma t h_{t}\left(i / 2^{m}\right)}\right\}\right] \\
& =\lim _{m \rightarrow \infty} \mathbb{P}\left[\exp \left\{-\sum_{i=-m 2^{m}}^{m 2^{m}} \frac{2 Z_{0}\left(\left[(i-1) / 2^{m}, i / 2^{m}[) h_{t}\left(i / 2^{m}\right)\right.\right.}{2+\gamma t h_{t}\left(i / 2^{m}\right)}\right\}\right] \\
& =\mathbb{P}\left[\exp \left\{-\left\langle Z_{0}, \frac{2 h_{t}}{2+\gamma t h_{t}}\right\rangle\right\}\right] \\
& =\mathbb{P}\left[\exp \left\{-\left\langle Z_{t}, h_{0}\right\rangle\right\}\right] .
\end{aligned}
$$

Therefore,

$$
Z_{t}^{(m)} \stackrel{D}{\rightarrow} Z_{t}
$$

Further, by the definition of $\left(x_{i}(t)\right)$ and the additive property for Feller's branching diffusions we obtain that

$$
Z_{t}^{(m)}=\sum_{i=-\infty}^{\infty} \sum_{\Pi_{i-1} \leqslant j / 2^{m}<\Pi_{i}} \eta_{j}^{(m)}(t) \delta_{x_{i}(t)} \stackrel{D}{\rightarrow} \sum_{i=-\infty}^{\infty} \eta_{i}(t) \delta_{x_{i}(t)} .
$$

Putting these together gives (5.6).

This interplay is remarkable. On one hand, $Z$ can be constructed using the Arratia flow; on the other hand, $Z$ tells us how an initial measure $Z_{0}$ is transported over time under both the Arratia flow and the branching. 


\section{A more general model}

As Steven Evans pointed out to me that the proof of Theorem 3.2 only uses the branching property of the Feller's diffusion. This suggests that we can replace the Feller's branching diffusion by the square of the Bessel processes (BESQ) to incorporate immigration. We are going to carry it out in this section.

For $x \geqslant 0$ and $\delta \geqslant 0$ the square of $\delta$-dimensional Bessel process starting at $x$, denoted by $\mathrm{BESQ}^{\delta}(x)$, is a nonnegative valued process $\xi$ which solves the following stochastic differential equation

$$
\xi_{t}=x+\int_{0}^{t} \sqrt{\gamma \xi_{s}} \mathrm{~d} B_{s}+\delta t,
$$

where $B$ is a one-dimensional Brownian motion.

Applying Itô's formula we obtain the following partial differential equation for $V(\lambda, t):=\mathbb{P}\left[\exp \left\{-\lambda \xi_{t}\right\}\right]$,

$$
\frac{\partial V}{\partial t}+\frac{\gamma \lambda^{2}}{2} \frac{\partial V}{\partial \lambda}+\delta \lambda V=0, \quad V(\lambda, 0)=\exp \{-\lambda x\}
$$

Then we can recover the Laplace transform for $\xi_{t}$ as

$$
\mathbb{P}\left[\exp \left\{-\lambda \xi_{t}\right\}\right]=\left(\frac{2}{2+\lambda \gamma t}\right)^{2 \delta / \gamma} \exp \left\{-\frac{2 \lambda x}{2+\lambda \gamma t}\right\} .
$$

Notice that the Feller's branching diffusion is just $\mathrm{BESQ}^{0}$. We refer to Chapter XI of [13] for a more detailed introduction on the Bessel processes.

It is easy to see from (6.1) that $\operatorname{BESQ}^{\delta}(x)$ is additive in both $\delta$ and $x$; i.e. if $\left\{\xi_{i}, i=1, \ldots, m\right\}$ is a collection of independent processes such that each $\xi_{i}$ is $\mathrm{BESQ}^{\delta_{i}}\left(x_{i}\right)$. Then $\sum_{i=1}^{m} \xi_{i}$ is $\mathrm{BESQ}^{\sum_{i=1}^{m} \delta_{i}}\left(\sum_{i=1}^{m} x_{i}\right)$.

Now we are going to modify the process $Z$ defined in Section 3 by letting the masses of the particles be governed by BESQs. Since the dimension is an additional parameter for BESQ, we need to introduce another measure-valued process $\Delta$ to describe the evolution of the dimension.

As in Section 3, we first consider two systems of interacting particles. Given $Z_{0} \in M_{F}(\mathbb{R})$, write $\bar{z}:=Z_{0}(\mathbb{R})$ and $\bar{Z}_{0}:=Z_{0} / \bar{z}$. For any $m$, choose $x_{1}, \ldots, x_{m}$ to be i.i.d. random variables with common distribution $\bar{Z}_{0}$. Given $\Delta_{0} \in$ $M_{F}(\mathbb{R})$, write $\bar{\delta}:=\Delta_{0}(\mathbb{R})$ and $\bar{\Delta}:=\Delta_{0} / \bar{\delta}$. Let $x_{1}^{\prime}, \ldots, x_{m}^{\prime}$ be i.i.d. random variables with common distribution $\bar{\Delta}$. Let $\left(X_{1}, \ldots, X_{m}, X_{1}^{\prime}, \ldots, X_{m}^{\prime}\right)$ be a $2 m$-dimensional coalescing Brownian motion starting at $\left(x_{1}, \ldots, x_{m}, x_{1}^{\prime}, \ldots, x_{m}^{\prime}\right)$.

Let $\left(\xi_{1}, \ldots, \xi_{m}\right)$ and $\left(\delta_{1}, \ldots, \delta_{m}\right)$ be two collections of $m$ independent processes such that $\xi_{i}$ is $\operatorname{BESQ}^{0}(\bar{z} / m)$ and $\delta_{i}$ is $\mathrm{BESQ}^{\bar{\delta} / m}(0)$. We further assume that $\left(x_{i}\right)$ and $\left(x_{i}^{\prime}\right)$ are independent, and $\left(X_{1}, \ldots, X_{m}, X_{1}^{\prime}, \ldots, X_{m}^{\prime}\right),\left(\xi_{i}\right)$ and $\left(\delta_{i}\right)$ are all independent.

Now we are ready to define the two $M_{F}(\mathbb{R})$-valued processes

$$
Z_{t}^{(m)}:=\sum_{i=1}^{m} \xi_{i}(t) \delta_{X_{i}(t)}+\sum_{i=1}^{m} \delta_{i}(t) \delta_{X_{i}^{\prime}(t)}
$$

and

$$
\Delta_{t}^{(m)}:=\sum_{i=1}^{m} \frac{\bar{\delta}}{m} \delta_{X_{i}^{\prime}(t)} .
$$

Similar to Lemma 3.1 we can show that both $\left\{Z^{(m)}\right\}$ and $\left\{\Delta^{(m)}\right\}$ are C-relatively compact in $D\left(M_{F}(\mathbb{R})\right)$. They have unique weak limits by Theorem 6.1 , which we will prove shortly.

Let $Z$ and $\Delta$ be the weak limits for $\left\{Z^{(m)}\right\}$ and $\left\{\Delta^{(m)}\right\}$. Intuitively, $\left\{\left(Z_{0}(B), \Delta_{0}(B)\right): B \in \mathcal{B}(\mathbb{R})\right\}$ describes the initial mass-dimension distribution on $\mathbb{R}$, and $\left\{\left(Z_{t}(B), \Delta_{t}(B)\right): B \in \mathcal{B}(\mathbb{R}), 0 \leqslant t<\infty\right\}$ describes the simultaneous mass-dimension evolution for such a model, which we call a supersquare of Bessel process with spatial coalescing Brownian motion. 
As before, write $\left(Y_{1}, \ldots, Y_{2 n}\right)$ for a $2 n$-dimensional coalescing Brownian motion starting at $\left(y_{1}, \ldots, y_{2 n}\right)$ with $y_{1} \leqslant \cdots \leqslant y_{2 n}$. For any nonnegative constants $a_{j}, b_{j}, j=1, \ldots, n$, and $t>0$, let $h_{t}$ be defined as in (3.2) and let

$$
\left.\left.h_{t}^{\prime}(\cdot):=\sum_{j=1}^{n} b_{j} 1\{] Y_{2 j-1}(t), Y_{2 j}(t)\right]\right\}(\cdot) \text {. }
$$

The next result determines the joint distribution for $\left(Z_{t}(B), \Delta_{t}(B)\right), B \in \mathcal{B}(\mathbb{R})$.

Theorem 6.1. For any $a_{j} \geqslant 0, b_{j} \geqslant 0, j=1, \ldots, n$, and any $y_{1} \leqslant \cdots \leqslant y_{2 n}$, we have

$$
\mathbb{P}\left[\exp \left\{-\left\langle Z_{t}, h_{0}\right\rangle-\left\langle\Delta_{t}, h_{0}^{\prime}\right\rangle\right\}\right]=\mathbb{P}\left[\exp \left\{-\left\langle Z_{0}, \frac{2 h_{t}}{2+\gamma t h_{t}}\right\rangle-\left\langle\Delta_{0}, \frac{2}{\gamma} \ln \left(1+\frac{\gamma t h_{t}}{2}\right)+h_{t}^{\prime}\right\rangle\right\}\right] .
$$

Proof. We might assume that $\left(Y_{i}\right)$ is independent of $\left(\xi_{i}\right)$ and $\left(\delta_{i}\right)$. To prove (6.2), again, we first fix $\left(\delta_{i}\right)$ and $\left(\xi_{i}\right)$. It follows from Theorem 2.1 that

$$
\begin{aligned}
\mathbb{P}\left[\exp \left\{-\left\langle Z_{t}^{(m)}, h_{0}\right\rangle-\left\langle\Delta_{t}^{(m)}, h_{0}^{\prime}\right\rangle\right\}\right] & =\mathbb{P}\left[\exp \left\{-\sum_{i=1}^{m} \xi_{i}(t) h_{0}\left(X_{i}(t)\right)-\sum_{i=1}^{m} \delta_{i}(t) h_{0}\left(X_{i}^{\prime}(t)\right)-\sum_{i=1}^{m} \frac{\bar{\delta} h_{0}^{\prime}\left(X_{i}^{\prime}(t)\right)}{m}\right\}\right] \\
& =\mathbb{P}\left[\exp \left\{-\sum_{i=1}^{m} \xi_{i}(t) h_{t}\left(x_{i}\right)-\sum_{i=1}^{m} \delta_{i}(t) h_{t}\left(x_{i}^{\prime}\right)-\sum_{i=1}^{m} \frac{\bar{\delta} h_{t}^{\prime}\left(x_{i}^{\prime}\right)}{m}\right\}\right] .
\end{aligned}
$$

We then fix $\left(x_{i}\right)$ and $\left(x_{i}^{\prime}\right)$, and take expectations with respect to $\left(\xi_{i}\right)$ and $\left(\delta_{i}\right)$. By (6.1) the right-hand side of (6.3) is equal to

$$
\begin{aligned}
& \mathbb{P}\left[\prod_{i=1}^{m}\left(\frac{2}{2+\gamma t h_{t}\left(x_{i}^{\prime}\right)}\right)^{2 \bar{\delta} /(\gamma m)} \exp \left\{-\frac{2 \bar{z} h_{t}\left(x_{i}\right)}{m\left(2+\gamma t h_{t}\left(x_{i}\right)\right)}-\frac{\bar{\delta} h_{t}^{\prime}\left(x_{i}^{\prime}\right)}{m}\right\}\right] \\
& \quad=\mathbb{P}\left[\left\langle\bar{Z}_{0}, \exp \left\{-\frac{2 \bar{z} h_{t}}{m\left(2+\gamma t h_{t}\right)}\right\}\right\}^{m}\left(\bar{\Delta}_{0},\left(1+\frac{\gamma t h_{t}}{2}\right)^{-2 \bar{\delta} /(\gamma m)} \exp \left\{-\frac{\bar{\delta} h_{t}^{\prime}}{m}\right\}\right\}^{m}\right],
\end{aligned}
$$

where we condition on $\left(Y_{i}\right)$ to obtain the equality.

Let $m \rightarrow \infty$ in (6.4). We finally have

$$
\begin{aligned}
\mathbb{P} & {\left[\exp \left\{-\left\langle Z_{t}, h_{0}\right\rangle-\left\langle\Delta_{t}, h_{0}^{\prime}\right\rangle\right\}\right] } \\
& =\lim _{m \rightarrow \infty} \mathbb{P}\left[\exp \left\{-\left\langle Z_{t}^{(m)}, h_{0}\right\rangle-\left\langle\Delta_{t}^{(m)}, h_{0}^{\prime}\right\rangle\right\}\right] \\
& =\lim _{m \rightarrow \infty} \mathbb{P}\left[\left\langle\bar{Z}_{0}, 1-\frac{2 \bar{z} h_{t}}{m\left(2+\gamma t h_{t}\right)}\right\rangle^{m}\left\langle\bar{\Delta}_{0},\left(1-\frac{2 \bar{\delta}}{\gamma m} \ln \left(1+\frac{\gamma t h_{t}}{2}\right)\right)\left(1-\frac{\bar{\delta} h_{t}^{\prime}}{m}\right)\right\rangle^{m}\right] \\
& =\lim _{m \rightarrow \infty} \mathbb{P}\left[\left\langle\bar{Z}_{0}, 1-\frac{2 \bar{z} h_{t}}{m\left(2+\gamma t h_{t}\right)}\right\rangle^{m}\left\langle\bar{\Delta}_{0},\left(1-\frac{2 \bar{\delta}}{\gamma m} \ln \left(1+\frac{\gamma t h_{t}}{2}\right)-\frac{\bar{\delta} h_{t}^{\prime}}{m}\right)\right\rangle^{m}\right] \\
& =\mathbb{P}\left[\exp \left\{-\left\langle\bar{Z}_{0}, \frac{2 \bar{z} h_{t}}{2+\gamma t h_{t}}\right\rangle\right\} \exp \left\{\left\langle\bar{\Delta}_{0}, \frac{2 \bar{\delta}}{\gamma} \ln \left(1+\frac{\gamma t h_{t}}{2}\right)+\bar{\delta} h_{t}^{\prime}\right\rangle\right\}\right] \\
& =\mathbb{P}\left[\exp \left\{-\left\langle Z_{0}, \frac{2 h_{t}}{2+\gamma t h_{t}}\right\rangle-\left\langle\Delta_{0}, \frac{2}{\gamma} \ln \left(1+\frac{\gamma t h_{t}}{2}\right)+h_{t}^{\prime}\right\rangle\right\}\right] .
\end{aligned}
$$

Remark 6.2. Notice that $\Delta$ is just the process $Z$ in Theorem 3.2 with $\gamma=0$.

The generalized model considered in this section will not die out if $\mu>0$. Many of the properties in Section 3 and Section 4 can be discussed in a similar fashion. But we leave the details to interested readers. 


\section{Acknowledgement}

The author is grateful to Steven Evans for a suggestion that results in Section 5 of this paper. The author thanks Carl Müller for a helpful comment. He also thanks an anonymous referee for very helpful comments.

\section{References}

[1] R. Arratia, Coalescing Brownian motions on the line, Ph.D. thesis, University of Wisconsin, Madison, 1979.

[2] R.W.R. Darling, Isotropic stochastic flows: a survey, in: M. Pinsky, M. Wihstutz (Eds.), Diffusion Processes and Related Problems in Analysis, vol. 2, Birkhäuser, Boston, 1992, pp. 75-94.

[3] D.A. Dawson, Z.H. Li, Construction of immigration superprocesses with dependent spatial motion from one-dimensional excursions, Probab. Theory Related Fields 127 (1) (2003) 37-61.

[4] D.A. Dawson, Z.H. Li, H. Wang, Superprocesses with dependent spatial motion and general branching densities, Electron. J. Probab. 6 (25) (2001) 1-33.

[5] D.A. Dawson, Z.H. Li, X. Zhou, Superprocesses with coalescing Brownian spatial motion as large scale limits, J. Theoret. Probab. 17 (3) (2004) 673-692.

[6] A.M. Etheridge, An Introduction to Superprocesses, University Lecture Series, vol. 20, Amer. Math. Soc., 2000.

[7] S.N. Ethier, T.G. Kurtz, Markov Processes: Characterization and Convergence, Wiley, New York, 1986.

[8] J. Jacod, A.N. Shiryaev, Limit Theorems for Stochastic Processes, Springer-Verlag, Berlin, 1987.

[9] O. Kallenberg, Random Measures, Academic Press, New York, 1976.

[10] Y. Le Jan, O. Raimond, Flows, coalescence and noise, Ann. Probab. 32 (2) (2005) 1247-1315.

[11] Z. Ma, K. Xiang, Superprocesses of stochastic flows, Ann. Probab. 29 (1) (2001) 317-343.

[12] E. Perkins, Dawson-Watanabe superprocesses and measure-valued diffusions, in: Lectures on Probability Theory and Statistics, Saint-Flour, 1999, in: Lecture Notes in Math., vol. 1781, Springer, Berlin, 2002, pp. 125-329.

[13] D. Revuz, M. Yor, Continuous Martingales and Brownian Motion, Springer, Berlin, 1991.

[14] G. Skoulakis, R.J. Adler, Superprocesses over a stochastic flow, Ann. Appl. Probab. 11 (2) (2002) 488-543.

[15] F. Soucaliuc, B. Tóth, W. Werner, Reflection and coalescence between independent one-dimensional Brownian paths, Ann. Inst. H. Poincaré Probab. Statist. 36 (4) (2000) 509-545.

[16] B. Tóth, W. Werner, The True self-repelling motion, Probab. Theory Related Fields 111 (3) (1997) 375-452.

[17] R. Tribe, The behavior of superprocesses near extinction, Ann. Probab. 20 (1) (1992) 286-311.

[18] J. Xiong, X. Zhou, On the duality between coalescing Brownian motions, Canad. J. Math. 57 (1) (2005) $204-224$. 